\title{
IQA-embedded fragment attributed molecular system energy change in exploring intramolecular interactions
}

\author{
Ignacy Cukrowski \\ Department of Chemistry, Faculty of Natural and Agricultural Sciences, University of Pretoria, \\ Lynnwood Road, Hatfield, Pretoria 0002, South Africa
}

E-mail: ignacy.cukrowski@up.ac.za

Landline: +27124203988 


\section{ABSTRACT}

Energy of intramolecular interaction cannot be measured experimentally. Deeply-rooted in the Interacting Quantum Atoms framework, expressions for a fragment attributed molecular system energy change (FAMSEC) are proposed and implemented to quantify energy contribution made by a molecular fragment $\mathscr{E}=\{A, B\}$ made of interacting atoms. A classical nature of (i) $\mathrm{N} \bullet \bullet \mathrm{H}$ (in protonated ethylenediamine, Hen) and $\mathrm{O} \bullet \bullet \mathrm{H}$ (in protonated ethanolamine, Hea) and (ii) $\mathrm{O} \bullet \bullet \mathrm{O}$ (in eclipsed glycol, gc) was fully recovered and their origin explored; $\mathrm{N} \bullet \bullet \mathrm{H}$ and $\mathrm{O} \bullet \bullet \mathrm{H}$ stabilize respective molecules locally, local-FAMSEC, and globally, mol-FAMSEC (opposite applies to $\mathrm{O} \bullet \bullet \mathrm{O}$ in $\mathbf{g c}$ ). Higher energy of planar biphenyl (bph) was attributed to (i) $\mathrm{C}$-atoms linking the rings due to an unfavorable change in interactions with all atoms of bph and (ii) increase in self-atomic energies of the remaining C-atoms of the bph bay. Considering orthohydrogens, they (i) do not conform to steric clash, (ii) resemble stabilizing interactions in Hen and Hea and (iii) follow changes in physical properties (on interaction formation) found for heteroatoms in Hen and Hea (opposite was found for O-atoms in gc). Moreover, the molFAMSEC term (i) accounts to some extent, although indirectly, for the geometric deformation energy of all atoms not involved in the intramolecular interaction, (ii) equally applies to any kind of (de)stabilizing or QTAIM (non)bonded interaction, and (iii) can equally be used for any size of a molecular fragment (e.g. functional groups) as well as for intermolecular interactions.

Keywords: Interacting Quantum Atom; Interacting Quantum Fragments; Chemical bond; Steric clash; Intramolecular interaction; Biphenyl. 


\section{Introduction}

Inter- and intramolecular interactions, e.g., hydrogen bonds, are extensively investigated [1,2] but their bond energy is not a physical observable, hence it is not directly measurable. One can gain a valuable insight on the nature of an intermolecular interaction by employing the energy and charge decomposition techniques [3-5] and stabilization of a molecule can be estimated as the difference between the energy of a complex and the energies of its isolated monomers [6-9]. It is important to realize, however, that even when the same kind of intermolecular H-bond is considered, e.g., $\mathrm{OH} \cdots \mathrm{O}$, one observes different energetic stabilization attributed to this interaction in different molecules which clearly indicates importance of the surrounding atomic environment. In other words, the computed difference between the energy of the adduct and the sum of the energies of the separate component molecules does not provide direct information on energy contribution, made only by atoms involved in the interatomic H-bond, to a molecular system.

It is generally accepted that the energy decrease of a conformer of a molecule with an intramolecular hydrogen bond, when compared with an energy of a H-bond-free conformer of this molecule, is related to the strength of a $\mathrm{X}-\mathrm{H} \cdots \mathrm{Y}$ interaction, which in turn depends on the nature and properties of the $\mathrm{X}$ - and $\mathrm{Y}$-atoms. Unfortunately, one is not able to take a full advantage of classical energy partitioning schemes [3-5] in exploring intramolecular bonds because the overall energy effect between two molecular fragments is largely dominated by the reconstruction of a broken covalent bond. Instead, a suitable reference molecule(s) is often used to estimate the intramolecular hydrogen bond energy from (i) the calculated barriers for internal rotations of the end groups [7], (ii) 'thermodynamic cycle' of transitions between different structures [10] or combination of conformational, isodesmic or reference-free methods [11] involving the geometry corrected method [12], the related rotamers method [13,14], and Espinosa's method [15]. To account for distant interactions of the H-bond region with the remainder of the system, dedicated parameters, $Q$ or $\lambda[16]$ and $\nabla_{\mathrm{rp}}$ [17], were used in the study 
of resonance-assisted hydrogen-bond cooperativity to describe $\pi$-electron delocalization in the $\pi$ electron spacer linking the proton-donating and proton-accepting parts of the H-bond [18]. Finally, to estimate the energy of intramolecular H-bond, the molecular tailoring approach was proposed involving addition/subtraction of the single point energies of tailored individual molecular fragments [19].

The presence of Bader's atomic interaction line (a bond path, BP, defined within the quantum theory of atoms in molecule, QTAIM [20]) points at the presence of an inter- or intramolecular interaction and, not surprisingly, topological properties at a bond critical point (BCP) were used to uncover the nature and compare the relative strength of the interactions $[8,9,15]$. It is well documented that the presence of a BP indicates a privileged exchange-correlation channel which is of a stabilizing nature [21]. However, besides classical H-bonds, there are different intramolecular interactions which might be either of stabilizing or destabilizing nature even when a $\mathrm{BP}$ is observed. This is because the local diatomic interaction might be overall destabilizing [22-28] when, e.g., electrostatic term of the interaction dominates and is of repulsive nature. Recently, a Non-covalent Interaction (NCI) method [29-31] (it is making use of the reduced density gradient which, in density functional theory, describes the deviation from a homogeneous electron distribution) was proposed to identify intramolecular 'attractive' (density accumulation) and 'repulsive' (density depletion) interactions. It is consistent with topological properties (electron density) at a bond critical point, but its major advantage is in the analysis of interactions when a bond path is not observed.

Clearly, a great effort has been made and numerous methods and approaches were developed to gain information about the nature and strength of intramolecular interactions but they still remain elusive, particularly when compared with the insight one can gain from an analysis of intermolecular interactions. Moreover, it appears that all the above mentioned methods are largely focused on the strength of an intramolecular interaction by estimating energetic contribution localized to the interatomic region between atoms involved in such interaction. 
Any bonded or non-bonded intramolecular interaction might be either (i) just an unavoidable feature of a rigid molecular structure (e.g., a steric contact between H-atoms in a planar biphenyl) or (ii) a result of a preferential conformational change to minimize the energy of a molecule due to formation of, e.g., a classical intramolecular H-bond. In principle, one should be able to computationally construct a suitable conformer, regardless whether higher or lower in energy, where such intramolecular interaction is absent. Hence, this interaction-free structure should be suitable as a reference state, ref, to monitor any change of interest when a final structure, fin, of this molecule with an intramolecular interaction is formed. Furthermore, to gain a deeper insight on the nature of intramolecular interaction between atoms $\mathrm{A}$ and $\mathrm{B}$ and to estimate the energy contribution made to the entire molecule by the molecular fragment $\mathcal{G}$ made of A and B atoms, it would be of great interest and fundamental importance to identify, quantify and understand all major atomic and interatomic energy changes taking place throughout a molecule when ref changes to fin. This is exactly the approach taken here and it comes from a simple realization that on any $3 \mathrm{D}$ change of a molecule, global (on a molecular scale) energy changes involving all atoms must take place. We propose here, deeply rooted in the IQA technique [32], a fragment attributed molecular system energy change (FAMSEC) concept and methodology which makes use of the IQA-defined one-body (atomic) and two-body (interatomic) components of the total molecular energy but as stressed by Francisco et al. [33] 'Both contributions actually include all the many-body interactions that result from a quantum mechanical calculation'. This relatively simple and of general purpose protocol (designed to study attractive or repulsive, bonded or non-bonded interactions, regardless if a BP is present or not) is tested here on four model molecules, protonated ethylenediamine (Hen), protonated ethanolamine (Hea), glycol (gc) and biphenyl (bph).

\section{Computational details}

All structures, except the eclipsed form of a glycol (where steric clash between O-atoms takes place) and planar form of bph (where steric clash between $\mathrm{H}$-atoms takes place) were optimized 
without constraints using Gaussian 09, Revision D.01 [34], at the MP2/6-311++G(d,p) level of theory. Structures of Hen, Hea and ge were computed in the simulated aqueous environment (PCM/UFF) whereas data related to bph were obtained in the gas phase. In some cases we have also used data obtained from the single point calculations (SPC) at a B3LYP/6-311++G(d,p) level performed on the MP2-optimized structures and this will be clearly stated in the text. The fin forms of gc (eclipsed) and bph (planar) were fully energy optimized with a relevant dihedral angle being constrained. When wavefunctions required for the QTAIM and IQA analyses were generated in Gaussian from the MP2-optimized structures then a keyword 'density=current' was used. Topological analysis, including molecular graph generation and calculation of all energy terms within the QTAIM and IQA frameworks, was carried out using Keith's AIMAll software [35] with default settings. Note that an accurate implementation of IQA requires well-defined second order density matrix but it is not implemented in AIMAll software for post HF levels of theory. Hence, the computed IQA-defined energy terms at B3LYP and MP2 levels are approximate due to the fact that the Müller approximation of the two-electron density matrix in terms of natural orbitals of the one-electron density matrix is used. However, the analysis of relative trends and the conclusions arrived at from this work should be considered as valid and representative as exemplified by full recovery of all characteristic properties of classical (de)stabilizing intramolecular interactions investigated in this work.

\section{Concept of the FAMSEC}

We want to achieve our main aim, a quantified energy contribution made to either a molecule or a molecular system by a new 3D placement of a selected molecular fragment, without breaking any existing 'structural' (covalent) bond. This immediately appeals to a direct use of the IQA-defined effective and net energies of a molecular fragment $[32,36]$, concepts which can be traced back to ideas coming from the theory of electronic separability of McWeeny [37] but were not used extensively within the IQA framework. To make it more descriptive for chemists at large and easier to link the use of a molecular fragment's effective and net energies with 
changes taking place throughout a molecule on an interaction formation, let us start with perfectly suited for the purpose IQA primary energy components $\left(E_{\text {add }}^{\mathrm{X}}, E_{\text {self }}^{\mathrm{X}}\right.$ and $\left.E_{\mathrm{int}}^{\mathrm{XY}}\right)[32]$.

The energy of a molecule, $E$, is partitioned into the additive atomic energy of any atom $\mathrm{X}$, $E_{\text {add }}^{\mathrm{X}}$, such that

$$
E=\sum_{\mathrm{X}} E_{\mathrm{add}}^{\mathrm{X}}
$$

and this is schematically presented in Fig. S1(a) of the SI. Furthermore, the additive atomic energy, say of an atom A in Fig. S1 of the SI, can be decomposed within the IQA framework to a self-atomic energy, $E_{\text {self }}^{\mathrm{A}}$ (a one-body component) and diatomic interaction energies between the atom $\mathrm{A}$ and any other atom $\mathrm{X}$ in a molecule, $E_{\mathrm{int}}^{\mathrm{AX}}$ (a two-body component) as described by Eq. 2; this energy partitioning is schematically represented in Fig. S1(b) of the SI.

$$
E_{\mathrm{add}}^{\mathrm{A}}=E_{\mathrm{self}}^{\mathrm{A}}+\sum_{\mathrm{X} \neq \mathrm{A}} 0.5 E_{\mathrm{int}}^{\mathrm{AX}}
$$

In other words, the energy $E$ of a molecule consists of all self-atomic and diatomic interaction energies

$$
E=\sum_{\mathrm{X}} E_{\mathrm{self}}^{\mathrm{X}}+0.5 \sum_{\mathrm{X}} \sum_{\mathrm{Y} \neq \mathrm{X}} E_{\mathrm{int}}^{\mathrm{XY}}
$$

where the $0.5 \sum_{\mathrm{X}} \sum_{\mathrm{Y} \neq \mathrm{X}} E_{\mathrm{int}}^{\mathrm{XY}}$ term is the energy contribution coming from all unique diatomic interactions within the molecular system, regardless whether atoms are covalently bonded or not.

When a molecule changes from a ref state (in principle (i) one is free to select any structure of a molecule which is most suitable for a particular purpose and this will become obvious from sections that follow and (ii) by using different ref states one can gain further insight on nature and underlying origin of an interaction) to fin state, most significant energy changes are expected to be observed among the diatomic interaction energies because of different relative to each other 3D placement of atoms. Variation in the self-atomic energies might be less significant provided that (i) the volume of atomic basin (hence the interatomic surface) does not change much, (ii) large charge transfer (out- or inflow of electrons from and to atomic basin) does not 
take place and (iii) electronic reorganization within atomic basin is negligible [32]. Because, besides quantifying changes in $E_{\mathrm{self}}^{\mathrm{X}}$ and $E_{\mathrm{int}}^{\mathrm{XY}}$ we also want to gain an additional insight on the A $\bullet \bullet B$ interaction, we will (i) express the interaction energy between the atoms separately and (ii) write all the energy expressions with clear focus on atoms A and B, to make notation as intuitive and self-explanatory as possible. At the same time, it will be shown how these expressions fall into more general IQA-defined energy terms, expressed in the molecular fragment notation, reported previously [32,36]; for convenience, a full set of applicable energy terms (symbols and equations) is included in Part 1 of the SI.

Let us now consider a molecule as consisting of two fragments, a fragment $G_{\text {made }}$ of two atoms $\mathrm{A}$ and $\mathrm{B}$ which are involved in, e.g., a classical intramolecular interaction in fin state of a molecular system and a fragment $\mathscr{H}$ which contains all the remaining atoms. Relative to the ref state of a molecular system, a self-atomic energy change of atoms constituting the fragment $\mathscr{Z}$ when in the fin state can be written as

$$
\Delta E_{\text {self }}^{\mathfrak{E}}=\Delta E_{\text {self }}^{\mathrm{A}}+\Delta E_{\text {self }}^{\mathrm{B}}=\left({ }^{\text {fin }} E_{\text {self }}^{\mathrm{A}}-{ }^{\text {ref }} E_{\text {self }}^{\mathrm{A}}\right)+\left({ }^{\mathrm{fin}} E_{\text {self }}^{\mathrm{B}}-{ }^{\text {ref }} E_{\text {self }}^{\mathrm{B}}\right)
$$

and this can be interpreted as a deformation energy of the fragment $\mathscr{Z}$, which is typically positive when atoms $\mathrm{A}$ and $\mathrm{B}$ are involved in a bonding interaction [32]. The combined change in the interaction energy, for the two atoms $\mathrm{A}$ and $\mathrm{B}$, with all other atoms in a molecule, i.e. atoms of the fragment $\mathscr{\Psi}$, can be expressed as

$$
\begin{aligned}
\Delta \sum_{\mathrm{X} \in \mathcal{F}} E_{\mathrm{int}}^{\mathrm{EX}} & ={ }^{\mathrm{fin}} \sum_{\mathrm{X} \in \mathcal{F}} E_{\mathrm{int}}^{\mathcal{E} \mathrm{X}}-{ }^{\mathrm{ref}} \sum_{\mathrm{X} \in \mathcal{F}} E_{\mathrm{int}}^{\mathcal{E X}} \\
& ={ }^{\mathrm{fin}}\left(\sum_{\mathrm{X} \in \mathscr{F}} E_{\mathrm{int}}^{\mathrm{AX}}+\sum_{\mathrm{X} \in \mathscr{F}} E_{\mathrm{int}}^{\mathrm{BX}}\right)-\left(\sum_{\mathrm{X} \in \mathscr{F}} E_{\mathrm{int}}^{\mathrm{AX}}+\sum_{\mathrm{X} \in \mathscr{F}} E_{\mathrm{int}}^{\mathrm{BX}}\right)
\end{aligned}
$$

which also represents a change in the interfragment interaction energy component, $\Delta E_{\text {int }}^{\mathscr{E} F}$, between atoms of fragment $\mathscr{G}$ and atoms of fragment $\mathscr{\Psi}[32]$. Note that the geometry of atoms $\mathrm{X} \in \mathscr{F}$ in the $r e f$ and $f i n$ states of a molecular system can also change and this might result in 
somewhat different (i) density distribution within atomic basins as well as (ii) net charge of these atoms. These two changes in the atoms' physical properties, in addition to their different 3D placement in ref and fin states, will also contribute to the computed change in the interaction energy term expressed in Eq. 5; hence, the geometric deformation energy of atoms not involved in the intramolecular interaction is, although indirectly, accounted for to some extent.

Even though atoms A and B might not be involved in an obvious, or classical, intramolecular interaction in ref (implying that their interatomic distance is larger than the sum of their van der Waals radii) they still interact with each other regardless of their placement. Because of that, we must account also for the change in the interaction energy between the two atoms,

$$
\Delta E_{\mathrm{int}}^{\mathrm{AB}}={ }^{\mathrm{fin}} E_{\mathrm{int}}^{\mathrm{AB}}-{ }^{\mathrm{ref}} E_{\mathrm{int}}^{\mathrm{AB}}
$$

which can also be seen as the change in the intrafragment interaction energy, $\Delta E_{\text {int }}^{\mathscr{E}}$. By combining the three energy terms (defined in Eqs. 4-6) we obtain the expression of interest

$$
E_{\mathrm{attr}-\mathrm{mol}}^{\mathscr{E}}=\Delta E_{\mathrm{self}}^{\mathscr{E}}+\Delta E_{\mathrm{int}}^{\mathrm{AB}}+\Delta \sum_{\mathrm{X} \in \mathscr{F}} E_{\mathrm{int}}^{\mathscr{E X}}
$$

which can also be written in more general fashion where any size of molecular fragments, say $\mathscr{M}$ and $\mathscr{N}$, can be considered, namely

$$
E_{\mathrm{attr}-\mathrm{mol}}^{\mathscr{M}}=\Delta E_{\mathrm{self}}^{\mathscr{M}}+\Delta E_{\mathrm{int}}^{\mathscr{M}}+\Delta E_{\mathrm{int}}^{\mathscr{M} \mathscr{T}} .
$$

where $\Delta E_{\text {int }}^{\mathscr{M}}$ accounts for the total intrafragment interaction energy between atoms of the fragment $\mathscr{M}$ whereas $\Delta E_{\text {int }}^{\mathscr{M P S}}$ stays for the interfragment interaction energy term, i.e., all diatomic interactions between atoms of $\mathscr{M}$ and atoms of $\mathscr{N}$.

Realizing that terms in Eq. 7 account for all (i) atoms in ref and fin states of a molecule, (ii) IQA-defined primary energy terms and (iii) energy contributions related to the atoms of the selected fragment $\mathscr{G}$, we interpret the sum of the three terms, $E_{\text {attr-mol }}^{\mathscr{E}}$, as an energy contribution the molecular fragment $\mathcal{G}_{\text {makes }}$ to an entire molecule when it changes from a ref to fin state (for brevity, we will call this energy term as a fragment attributed molecular system energy change, 
mol-FAMSEC). Furthermore, the sum of first two terms in Eq. 7 accounts for bonding and non-

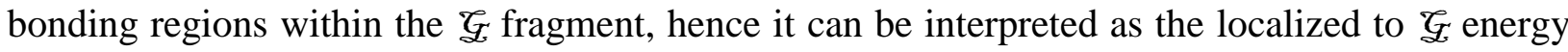
change, $E_{\text {attr-loc }}^{\mathscr{E}}($ local-FAMSEC) a molecule experiences when going from the ref to fin state,

$$
E_{\mathrm{attr}-\mathrm{loc}}^{\mathscr{E}}=\Delta E_{\mathrm{self}}^{\mathscr{E}}+\Delta E_{\mathrm{int}}^{\mathrm{AB}}
$$

Hence, Eq. 7 can be written as the sum of two components,

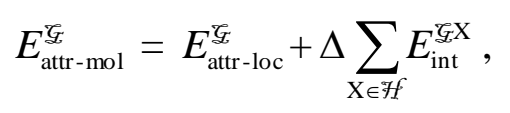

which, in more general form, can be written as $E_{\text {attr-mol }}^{\mathscr{M}}=E_{\text {attr-loc }}^{\mathscr{M}}+\Delta E_{\text {int }}^{\mathscr{M} \mathscr{T}}$.

The energy of a molecule can also be recovered by the use of energy terms defined for molecular fragments [32] and it is possible to show, see Part 2 of the SI, that the local and global energy changes can be expressed as differences in the net and effective energies of molecular fragment, $E_{\text {attr-loc }}^{\mathscr{E}}=\Delta E_{\text {net }}^{\mathscr{E}}$ and $E_{\text {attr-mol }}^{\mathscr{E}}=\Delta E_{\text {eff }}^{\mathscr{E}}$, respectively.

Finally, it is important to stress that $E_{\text {attr-mol }}^{\mathscr{E}}$ must not be confused with either (i) the change in the electronic molecular energies when going from ref to fin, $\Delta E={ }^{\text {fin }} E-{ }^{\text {ref }} E$, or (ii) the strength of an intramolecular interaction between $\mathrm{A}$ and $\mathrm{B}$. As a matter of fact, the $E_{\text {attr-mol }}^{\mathscr{E}}$ term might and, in principle, should be significantly different than $\Delta E$ because the mol-FAMSEC term does not account for all energy changes in a molecule. However, if the focus is on a leading intramolecular interaction involving atoms of a selected $\mathscr{E}$ fragment, then one might expect $E_{\text {attr-mol }}^{\mathscr{E}}=\Delta E_{\text {eff }}^{\mathscr{E}}>|\Delta E|$. Also, because one is free to probe any fragment to test its contribution to the molecular energy change, it is reasonable to expect mol-FAMSEC $\ll \Delta E$ in some instances. By 'interrogating' the specified three energy contributions, $\Delta E_{\text {self }}^{\mathscr{G}}, \Delta E_{\mathrm{int}}^{\mathrm{AB}}=\Delta E_{\mathrm{int}}^{\mathscr{G}}$ and $\Delta \sum_{\mathrm{X} \in \mathscr{F}} E_{\mathrm{int}}^{\mathscr{G} \mathrm{X}}=$ $\Delta E_{\text {int }}^{\text {EF }}$, we hope to gain an important insight on their significance, hence the origin and predominant physical nature of the mol-FAMSEC $=\Delta E_{\text {eff }}^{\complement}$ and local-FAMSEC $=\Delta E_{\text {net }}^{\mathscr{E}}$ terms. 


\subsection{Computationally affordable mol-FAMSEC expressions}

It is important to stress that the use of Eq. 7 requires computationally expensive IQA calculations for all atoms in a molecule. Then one might question the suitability of the FAMSEC concept for the study of large(r) systems (note that only small molecules were investigate by IQA to date). Just as an example, there are 231 unique diatomic interactions (all two-body and computationally most time-consuming components) in the investigated here biphenyl which is made of 22 atoms; hence, using the ref state of bhp requires computing 462 diatomic interactions to gain an insight on a single $\mathrm{H}--\mathrm{H}$ contact from the FAMSEC perspective which can be seen as computationally unaffordable.

However, as shown in details in Part 3 of the SI, it is possible to obtain $E_{\text {attr-mol }}^{\mathscr{E}}$ either from Eq. 11

$$
E_{\text {attr-mol }}^{\mathscr{E}}=2 \Delta E_{\text {add }}^{\mathscr{E}}-\Delta E_{\text {self }}^{\mathscr{E}}-\Delta E_{\text {int }}^{\mathrm{AB}}
$$

or from Eq. 12 (for details see Part 4 of the SI)

$$
E_{\mathrm{attr}-\mathrm{mol}}^{\mathscr{G}}=\Delta E_{\mathrm{self}}^{\mathscr{G}}-\Delta E_{\mathrm{int}}^{\mathrm{AB}}+\Delta \sum_{\mathrm{X} \neq \mathrm{A}} E_{\mathrm{int}}^{\mathrm{AX}}+\Delta \sum_{\mathrm{X} \neq \mathrm{B}} E_{\mathrm{int}}^{\mathrm{BX}}
$$

Note that Eqs 11 and 12 involve just two atoms of interest (the latter equation provides all the energy terms seen in Eq. 7), hence, now they can be computed economically.

Recalling that (i) the additive and self-atomic energies are always large and negative and (ii) the $\sum_{\mathrm{X} \neq \mathrm{A}} 0.5 E_{\mathrm{int}}^{\mathrm{AX}}$ values are always negative for any selected atom $\mathrm{A}$, a direct interpretation of these energy terms might not be straightforward. However, by monitoring changes taking place throughout a molecule (when going from its ref to fin state) one might gain an invaluable insight on the origin and significance of changes observed when, e.g., understanding of intramolecular interactions is of interest. This is exactly the approach taken here which makes use of groupedon-purpose IQA-defined energy terms. Obviously, a full IQA exploration of molecules is often not a feasible option. But, as demonstrated above, regardless of the size of a molecule, it is still possible to obtain all energy contributions in the mol-FAMSEC term by performing the IQA 
calculations only on two selected atoms. Consequently, this makes investigation of intramolecular bonds and interactions in large(r) molecules feasible.

\section{Results and discussions}

We decided to explore both, (de)stabilizing, interactions in selected molecules, namely: protonated ethylenediamine (Hen), protonated ethanolamine (Hea), glycol (gc) and biphenyl (bph). These molecules are presented, as molecular graphs, in Table 1 , where $\Delta E={ }^{\text {fin }} E-{ }^{\mathrm{ref}} E$, interatomic distances between atoms involved in close contacts and, when applicable, electron densities at a BCP are also shown. In general, the selection of molecular systems used can be seen as comprising two sets of molecules which classically, when just an interatomic distance is considered, could be classified as characterized by a presence of intramolecular (i) stabilizing $\mathrm{H} 11 \bullet{ }^{2} 7$ and $\mathrm{H} 12 \cdots \mathrm{O} 7$ interactions in Hen and Hea, respectively, in the first set of molecules, and (ii) destabilizing $\mathrm{O} 7 \bullet \bullet \mathrm{O} 9$ and $\mathrm{H} 9 \bullet \bullet \cdot \mathrm{H} 16$ interactions caused by steric clashes in $\mathbf{g c}$ and bph, respectively, in the second set. One must note that significantly smaller than the sum of the van der Waals radii interatomic distances are observed between atoms of all molecular fragments $Z_{\text {G }}$ marked with an oval in Table 1; all remaining atoms of a molecule constitute a molecular fragment $¥$. The relevant and intramolecular-interaction-free ref structures of these molecules are shown in Fig. S3 in the SI.

From the QTAIM perspective, however, molecules in each set differ in that a BP is present only in one of the molecules, Hen and bph. Because of that, when proposed by Koch and Popelier [38] topological criteria were to be used, the H12 $\bullet$ O7 interaction in Hea would not be characterized as a true intramolecular H-bond. Furthermore, interactions (contacts) in Hea and gc cannot be characterized and their energies quantified using topological properties as a bond path and associated BCP is not present.

Considering Hen and Hea, we observe a comparable decrease in the molecular energy, when going from ref to fin, by -4.1 and $-3.5 \mathrm{kcal} \mathrm{mol}^{-1}$, respectively. Typically, one attributes the 
resultant $\Delta E$ to the presence of a stabilizing intramolecular interaction. By analogy, the O--O and $\mathrm{H}-\mathrm{-H}$ contacts in the fin forms of $\mathbf{g c}$ and $\mathbf{b p h}$ might be linked with the increase in $\Delta E$ by +7.5 and $+4.5 \mathrm{kcal} \mathrm{mol}^{-1}$, respectively, hence classically they might be interpreted as destabilizing a molecule steric hindrance even though a BP is observed between ortho- $\mathrm{H}$ in the planar bph.

\section{Table 1}

Molecular graphs of fin structures of molecules investigated in this work. ${ }^{a}$

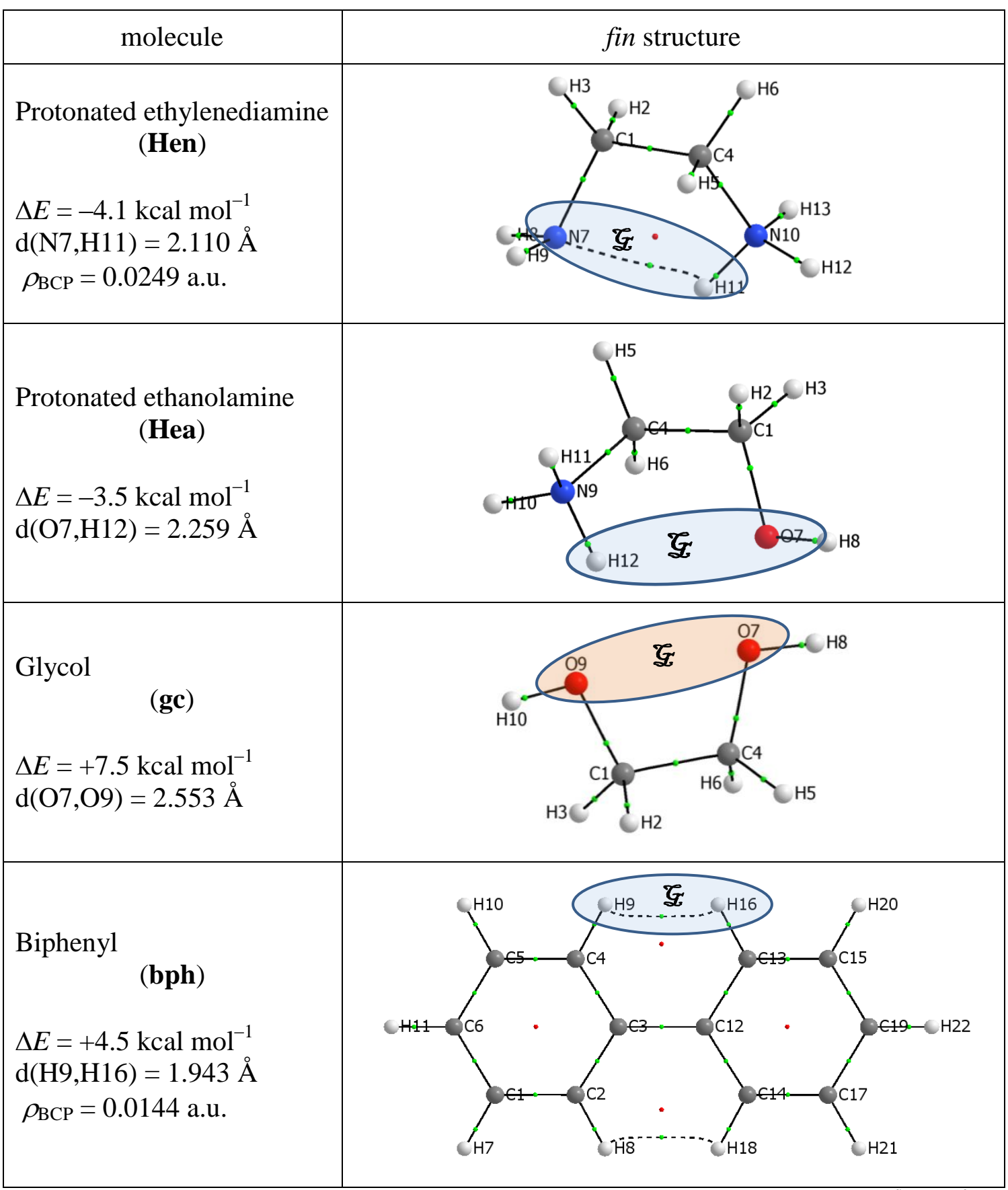

${ }^{\text {a }}$ All values were obtained at the MP2 level. $\Delta E$ is the difference in molecular electronic energies, ${ }^{\text {fin }} E-{ }^{\text {ref }} E$, where ref structures of the molecules are shown in Fig. S3 in the SI. K stands for molecular fragments (marked with an oval) containing atoms involved in intramolecular interactions examined in this work. 


\subsection{Exploring the changes in the additive and self-atomic energies}

The MP2 values of $E_{\text {add }}^{\mathrm{X}}$ and $E_{\text {self }}^{\mathrm{X}}$ for each atom X in both conformers of Hen, Hea, gc and for selected atoms in bph are shown in Tables S1-S4 in the SI. To gain further insight into trends in changes of atomic energies in $\mathbf{b p h}$, we performed IQA calculations for all atoms. To this effect, needed wavefunctions obtained from the SPC at the B3LYP/6-311++G(d,p) level of theory on the MP2-optimized fin (planar) and ref (twisted) structures of bph were used and the relevant results, $\Delta E_{\text {add }}^{\mathrm{X}}$ and $\Delta E_{\text {self }}^{\mathrm{X}}$, are shown in Table S5 in the SI. Relative to appropriate ref molecule changes in the atomic energies are shown in Table 2; note, that due to molecular symmetry in $\mathbf{g c}$ and $\mathbf{b p h}$, only values for selected atoms are included.

\subsubsection{Analysis concerning Hen and Hea}

Among all atoms, the largest variations in the $E_{\text {add }}^{\mathrm{X}}$ and $E_{\text {self }}^{\mathrm{X}}$ values are observed for $\mathrm{N} 7$ and $\mathrm{O} 7 ; E_{\text {add }}^{\mathrm{X}}$ decreased whereas $E_{\text {self }}^{\mathrm{X}}$ increased. Changes in the relevant atomic energies of the Hatoms participating in these intramolecular interactions, H11 in Hen and H12 in Hea, follow similar trend but the increase in $E_{\text {self }}^{\mathrm{X}}$ is larger than the decrease in their additive atomic energies.

Considering functional groups, we note that significant changes are also observed for atoms which are not directly involved in the intramolecular contact. For comparison and illustration, the observed changes in the atomic energies of atoms constituting functional groups in Hen and Hea are schematically shown in Fig. 1 where inner and outer circles represent $\Delta E_{\text {self }}^{\mathrm{X}}$ and $\Delta E_{\text {add }}^{\mathrm{X}}$, respectively, and the energy changes are color-coded (blue $=$ decrease, brown $=$ increase) with lighter color used for self-atomic energy. Remarkably, trends in the $\Delta E_{\text {self }}^{\mathrm{X}}$ and $\Delta E_{\text {add }}^{\mathrm{X}}$ values for relevant atoms of functional groups in both molecules are exactly the same even though different kind of intramolecular interaction is formed, e.g., $E_{\text {self }}^{\mathrm{X}}$ and $E_{\text {add }}^{\mathrm{X}}$ decreased for H12/H13 (in Hen) and H10/H11 (in Hea) whereas opposite trend in atomic energies is observed for H8/H9 (in Hen) and $\mathrm{H} 8$ in Hea. 
Table 2

Relative to a relevant reference molecule changes in the additive and self-atomic energies. ${ }^{\text {a }}$

\begin{tabular}{|c|c|c|c|c|}
\hline atom $\mathrm{X}$ & $\Delta E_{\mathrm{add}}^{\mathrm{X}}$ & atom X & $\Delta E_{\text {add }}^{\mathrm{X}}$ & $\Delta E_{\mathrm{self}}^{\mathrm{X}}$ \\
\hline \multicolumn{3}{|c|}{ Hen } & \multicolumn{2}{|l|}{ Hea } \\
\hline $\mathrm{C} 1$ & -0.9 & $-4.7 \mathrm{C} 1$ & -0.7 & -6.0 \\
\hline $\mathrm{H} 2$ & 1.0 & $0.2 \mathrm{H} 2$ & 1.0 & 0.2 \\
\hline $\mathrm{H} 3$ & 1.6 & $0.3 \mathrm{H} 3$ & 2.8 & 0.6 \\
\hline $\mathrm{C} 4$ & -1.3 & $-0.4 \mathrm{C} 4$ & -3.1 & -2.2 \\
\hline H5 & -0.9 & -0.3 H5 & 0.4 & -0.4 \\
\hline H6 & 0.1 & -0.4 H6 & -0.3 & -0.2 \\
\hline N7 & -19.2 & $13.3 \mathrm{O} 7$ & -12.4 & 6.1 \\
\hline H8 & 5.3 & $3.0 \mathrm{H} 8$ & 5.1 & 1.5 \\
\hline H9 & 4.8 & $1.6 \mathrm{~N} 9$ & 6.9 & 2.3 \\
\hline N10 & 8.2 & $9.2 \mathrm{H} 10$ & -3.4 & -1.4 \\
\hline H11 & -2.6 & $8.6 \mathrm{H} 11$ & -3.0 & -1.1 \\
\hline H12 & -5.9 & $-2.1 \mathrm{H} 12$ & -2.8 & 3.7 \\
\hline H13 & -5.1 & -1.8 & & \\
\hline \multicolumn{3}{|c|}{ gc } & \multicolumn{2}{|l|}{ bph } \\
\hline O9 & 9.6 & -0.3 C3 & -0.7 & -3.5 \\
\hline H10 & -3.0 & $-1.9 \mathrm{C} 4$ & 1.1 & 0.9 \\
\hline $\mathrm{C} 4$ & -1.4 & -2.5 H9 & -0.3 & 1.7 \\
\hline H3 & -0.1 & 0.5 & & \\
\hline H6 & -0.1 & 0.5 & & \\
\hline
\end{tabular}
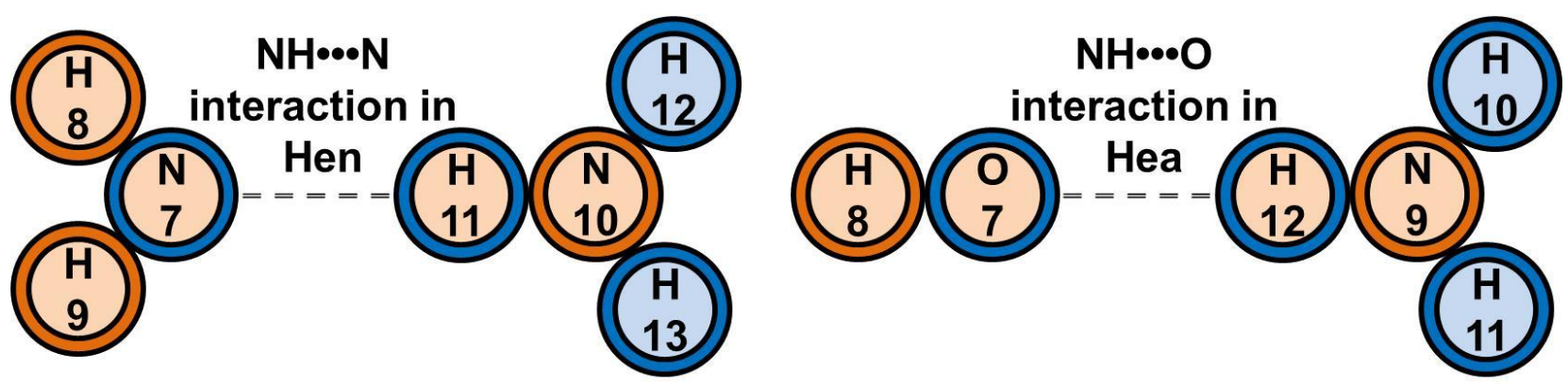

Fig. 1. A schematic representation of color-coded changes (blue $=$ decrease, brown $=$ increase) in atomic energies of indicated atoms in fin forms of Hen and Hea. Inner circle represents $\Delta E_{\text {self }}^{\mathrm{X}}$ (lighter color) and outer circle represents $\Delta E_{\text {add }}^{\mathrm{x}}$ (darker color).

Considering formation of intermolecular $\mathrm{AH} \cdot \bullet \mathrm{B}$ interactions, it is now well-documented (from the use of QTAIM-defined topological properties) that H-atoms are destabilized because their total virial-based atomic energy increases in molecules at the equilibrium geometry; this observation is used as one of the topological criteria to identify true H-bonds [38]. At the same 
time, the $\mathrm{A}$ and $\mathrm{B}$ atoms of the $\mathrm{AH} \bullet \cdot \mathrm{B}$ interaction are stabilized when compared with relevant values in monomers [39].

It was then of interest to find out if the same trend is observed when intramolecular interaction is formed; QTAIM- and IQA-defined atomic energies for all atoms of the functional groups $\left(-\mathrm{NH}_{2},-\mathrm{NH}_{3}{ }^{+}\right.$and $\left.-\mathrm{OH}\right)$ in Hen and Hea are shown in Table 3. Note that each $\Delta E^{\mathrm{X}}$ value (a change in the approximation to a virial-based total energy of an atom) was obtained by scaling the $T^{\mathrm{X}}$ value (the electronic kinetic energy of an atom) to obtain $E^{\mathrm{X}}$ expected for virial ratio equal exactly to two (the full molecular virial ratio obtained from optimization was $\approx 2.0013$ ). The kinetic energy $T^{\mathrm{X}}$ of (i) $\mathrm{H} 11$ and $\mathrm{H} 12$ decreased by about -9 and $-3 \mathrm{kcal} \mathrm{mol}^{-1}$ in Hen and Hea, respectively, (ii) N10 and N7 in Hen increased by about 16-17 kcal mol ${ }^{-1}$ and (iii) $\mathrm{N} 9$ and $\mathrm{O} 7$ in Hen increased by about $6 \mathrm{kcal} \mathrm{mol}^{-1}$. Hence, when the atomic virial theorem, $T^{\mathrm{X}}$ $=-E^{\mathrm{X}}$, is applied (it is commonly used in the QTAIM studies) then the H-atoms of the AH・••B interaction are destabilized (their $E^{\mathrm{H}}$ has increased) whereas the $\mathrm{A}$ and $\mathrm{B}$ atoms are stabilized.

\section{Table 3}

Relative to ref structures, changes in QTAIM- and IQA-defined atomic energies of atoms constituting functional groups in Hen and Hea. ${ }^{a}$

\begin{tabular}{llrrr}
\hline $\begin{array}{l}\text { Functional } \\
\text { group }\end{array}$ & Atom $\mathrm{X}$ & $\Delta E_{\text {self }}^{\mathrm{X}}$ & \multicolumn{1}{c}{$\Delta T^{\mathrm{X}}$} & \multicolumn{1}{c}{$\Delta E^{\mathrm{X}}$} \\
\hline \multicolumn{4}{c}{ Hen } \\
$-\mathrm{NH}_{2}$ & $\mathrm{~N} 7$ & 13.30 & 16.01 & -12.83 \\
& $\mathrm{H} 8$ & 2.98 & -4.81 & 4.84 \\
& $\mathrm{H} 9$ & 1.57 & -2.95 & 2.98 \\
$-\mathrm{NH}_{3}{ }^{+}$ & $\mathrm{N} 10$ & 9.16 & 17.50 & -14.32 \\
& $\mathrm{H} 11$ & 8.56 & -9.28 & 9.32 \\
& $\mathrm{H} 12$ & -2.14 & 2.99 & -2.97 \\
- & $\mathrm{H} 13$ & -1.84 & 2.69 & -2.67 \\
- & & Hea & & \\
$-\mathrm{OH}^{-}$ & $\mathrm{O} 7$ & 6.06 & 6.54 & -4.57 \\
& $\mathrm{H} 8$ & 1.55 & -2.51 & 2.52 \\
$-\mathrm{NH}_{3}{ }^{+}$ & $\mathrm{N} 9$ & 2.31 & 6.30 & -4.86 \\
& $\mathrm{H} 10$ & -1.36 & 1.60 & -1.59 \\
& $\mathrm{H} 11$ & -1.08 & 1.42 & -1.41 \\
& $\mathrm{H} 12$ & 3.71 & -3.14 & 3.15 \\
\hline
\end{tabular}

${ }^{\text {a }}$ Energies are in kcal $\overline{\mathrm{mol}^{-1} \text { at MP2; } E_{\mathrm{self}}^{\mathrm{X}}=T^{\mathrm{X}}+V_{\mathrm{ne}}^{\mathrm{XX}}+V_{\mathrm{ee}}^{\mathrm{XX}} \text {, by definition; } T^{\mathrm{X}}}$ - the electronic kinetic energy of an atom (a Hamiltonian form); $E^{\mathrm{X}}$ - approximation to a virial-based total energy of atom $\mathrm{X}$; 
Also, as found from the IQA studies of intermolecular H-bonds [39], the self-atomic energies of all atoms in the $\mathrm{AH} \bullet \bullet \mathrm{B}$ interaction increase and we observe exactly the same trend in both molecules. Data in Table 3 clearly shows that our results compare well with QTAIM- and IQAbased interpretation when trends in atomic energy changes of atoms participating in the $\mathrm{AH} \bullet \bullet \mathrm{B}$ interaction are concerned. Furthermore, we note that there is no difference in the trends of atomic energy changes between the two intramolecular interactions in Hen and Hea even though the latter has no BP.

Broadening the analysis to functional groups, we observe that all atoms of $-\mathrm{NH}_{3}{ }^{+}$in $\mathbf{H e n}$ and Hea, but $\mathrm{H}$-atoms involved in the interactions, are stabilized (their $E^{\mathrm{X}}$ decreased) but, as discussed above, different trends in these atoms $E_{\text {add }}^{\mathrm{X}}$ and $E_{\text {self }}^{\mathrm{X}}$ values are observed. The increase in the $E_{\text {self }}^{\mathrm{X}}$ values is expected [33] when a significant rearrangement in the electron density within atomic basins is observed upon the formation of a bonding interaction. Hence, we have inspected changes in net charges of atoms of the functional groups as well as $-\mathrm{CH}_{2}-$ fragments see Fig. 2 where +/- indicates an increase/decrease in the electron population.
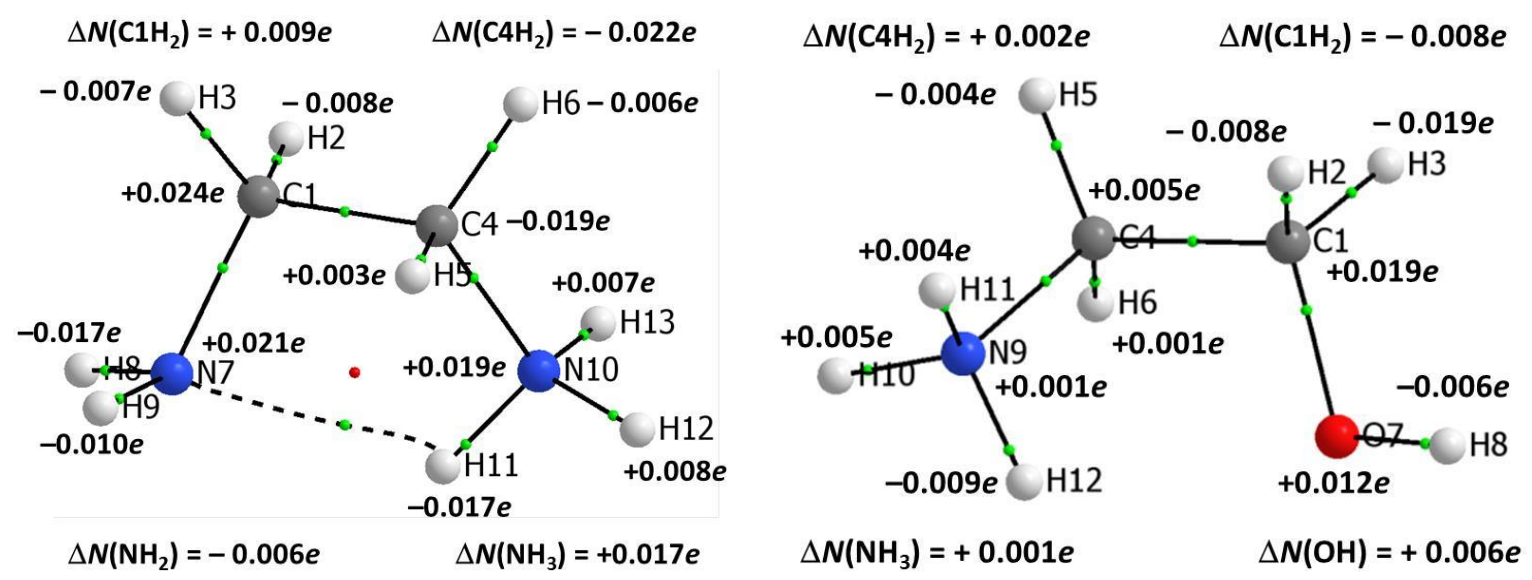

Fig. 2. Change (at MP2) in the electron population of atoms in Hen and Hea; minus indicates an outflow and plus an inflow of electrons on the interaction formation.

A large electron rearrangement took place throughout the molecules but the general pattern of the electron in- and outflow is very much the same in both molecules. Considering atoms directly involved in the intramolecular interactions, we observe a large inflow of electrons to 
heteroatoms $(+21 \mathrm{~m} e$ for $\mathrm{N} 7$ in Hen and $+12 \mathrm{me}$ for $\mathrm{O} 7$ in Hea) which is accompanied by a somewhat smaller outflow of electrons from $\mathrm{H}$-atoms $(-17 \mathrm{~m} e$ for $\mathrm{H} 11$ in Hen and $-9 \mathrm{~m} e$ for H12 in Hea). In the case of remaining atoms of the functional groups we note that (i) an inflow of electrons is observed for $\mathrm{N}$ - and $\mathrm{H}$-atoms of the $-\mathrm{NH}_{3}{ }^{+}$groups in both molecules, (ii) an outflow of electrons took place for $\mathrm{H}$-atoms of the $-\mathrm{NH}_{2}$ and $-\mathrm{OH}$ groups, and (iii) changes observed are more significant in Hen where a BP is observed.

All these changes facilitate an increase in charge polarization between atoms directly involved which must result in strengthening of the electrostatic component of the interactions; we observe differentiation in the charge of 38me between N7 and H11 in Hen and 21me between $\mathrm{O} 7$ and $\mathrm{H} 12$ in Hea. Interestingly, also $\mathrm{C} 1$ atoms of both molecules (they are covalently bonded to heteroatoms involved in the intramolecular interactions) experience a large inflow of electrons. As a matter of fact, this gain in electron population is the largest among all atoms in both molecules. Moreover, it appears that an increase in electron population correlates well with a decrease in $E^{\mathrm{X}}$ of atoms of functional groups in Hen and Hea, but it cannot be easily correlated with variation in $E_{\text {self }}^{\mathrm{X}}, e . g$, (i) $E_{\text {self }}^{\mathrm{X}}$ of all atoms increased in the $-\mathrm{NH}_{2}$ group of $\mathbf{H e n}$ even though a gain in electrons took place only for the $\mathrm{N}$-atom, or (ii) an increase in $E_{\text {self }}^{\mathrm{X}}$ is observed for all atoms directly involved in the interactions in Hen and Hea but only heteroatoms became more negative.

These observations prompted us to explore a broader range of properties and we found many trends holding for all atoms in Hen and Hea - see Table S6 and associated text in the SI - some of trends will be discussed in following sections.

\subsubsection{Analysis concerning gc}

Focusing on O-atoms, which are involved in a steric clash in the fin (eclipsed) form of gc, their $\Delta E_{\text {add }}^{\mathrm{O}}$ values are the largest among all atoms and they increased but, interestingly, their $E_{\text {self }}^{\mathrm{O}}$ hardly changed (see Table 2). The increase in the $E_{\text {add }}^{\mathrm{O}}$ values is accompanied by a 
significant outflow of electrons and this might be rationalized in terms of minimizing repulsion between $\mathrm{O} 7$ and $\mathrm{O} 9$. Their electron population decreased by $-19 \mathrm{me}$ and is mainly and quite evenly distributed among all $\mathrm{H}$-atoms of a molecule; a relative change in the electron population in fin form of ge is shown in Fig. 3.

Because the nature of the O--O interaction in the forced-to-be eclipsed form of gc is very much different, when compared with spontaneous formation of the lowest energy conformers of Hen and Hea, it was of interest to find out whether trends found from the analysis of data in Table S6 in the SI are also reproduced in gc. Analysis of data shown in Table S7 in the SI reveals significant differences and we note, e.g., that the trend-1 (when $\Delta \operatorname{Vol}^{\mathrm{X}}<0$ and $\Delta N^{\mathrm{X}}<0$ then $\Delta E_{\text {self }}^{\mathrm{X}}>0, \Delta E^{\mathrm{X}}>0$ and $\Delta V_{\mathrm{ne}}^{\mathrm{XX}}>0$ ) found for Hen and Hea does not hold for O-atoms for which a decrease in $E_{\text {self }}^{\mathrm{X}}$ takes place, or the trend-2 (when $\Delta V o l^{\mathrm{X}}>0$ and $\Delta N^{\mathrm{X}}>0$ then $\Delta E_{\text {self }}^{\mathrm{X}}<$ $0, \Delta E^{\mathrm{X}}<0$ and $\left.\Delta V_{\mathrm{ne}}^{\mathrm{XX}}<0\right)$ holds for atoms of the functional -OH group but does not apply to Catoms for which changes in opposite directions for $E^{\mathrm{X}}$ and $V_{\mathrm{ne}}^{\mathrm{XX}}$ are observed.
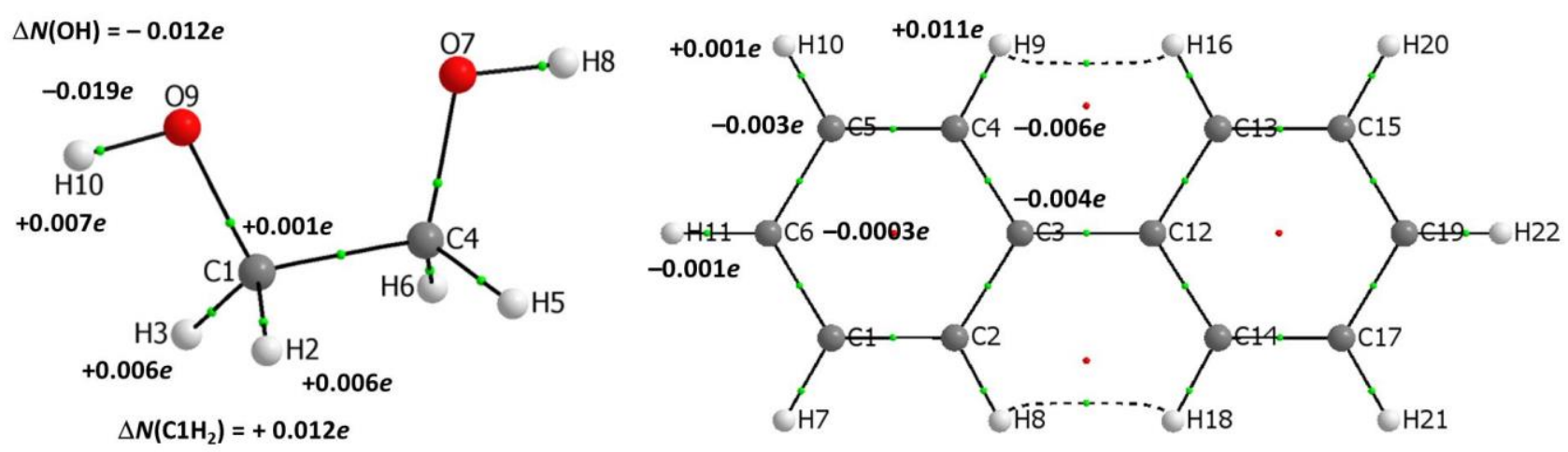

Fig. 3. Relative to the relevant ref structure changes (at MP2) in the electron population of atoms in the fin forms of $\mathbf{g c}$ and $\mathbf{b p h}$; minus indicates an outflow and plus an inflow of electrons on the interaction formation.

Furthermore, the set of the following indices, $\Delta E_{\text {self }}^{\mathrm{X}}<0, \Delta E_{\text {add }}^{\mathrm{X}}>0, \Delta T^{\mathrm{X}}<0, \Delta V_{\mathrm{ne}}^{\mathrm{XX}}>0$, $\Delta V_{\mathrm{ee}}^{\mathrm{XX}}<0, \Delta N^{\mathrm{X}}<0$ and $\Delta V \operatorname{Vol}^{\mathrm{X}}<0$, which was obtained for $\mathrm{O}$-atoms involved in steric clash in gc is (i) not observed among atoms of functional groups in Hen and Hea, and (ii) opposite to set(A) of indices found for the heteroatoms N7 (in Hen) and O7 (in Hea) - see Table S6 in the SI. 
Interestingly, there appears to be a correlation in gc which does not exist in Hen and Hea, namely when $\Delta V \operatorname{Vol}^{\mathrm{X}}>0$ then $\Delta d_{\mathrm{e}}^{\mathrm{X}}<0$ (and vice versa) holds for all atoms.

Intuitively, the observed different trends in atomic properties might be rationalized in terms of totally different nature of the intramolecular interactions in gc, Hen and Hea. However, to attempt a fully supported and in depth analysis of these trends on a fundamental level would be premature at this stage; much larger population of (de)stabilizing interactions in different molecules (hence different atomic environment) is required.

\subsubsection{Analysis concerning bhp}

A very different picture, and, in general, opposite to what is observed in $\mathbf{g c}$, emerges from the analysis of $\mathbf{H}$-atoms involved in the steric hindrance in the planar form of $\mathbf{b p h}-$ see Fig. 4 where the same schematic representation in atomic energy changes is used as described in details for Fig. 1. The $E_{\text {add }}^{\mathrm{H}}$ values of ortho-hydrogens (see Table 2) changed marginally in a stabilizing manner, $\Delta E_{\text {add }}^{\mathrm{H}}=-0.3 \mathrm{kcal} \mathrm{mol}^{-1}$, but their self-atomic energies increased as observed for interactions in Hen and Hea.
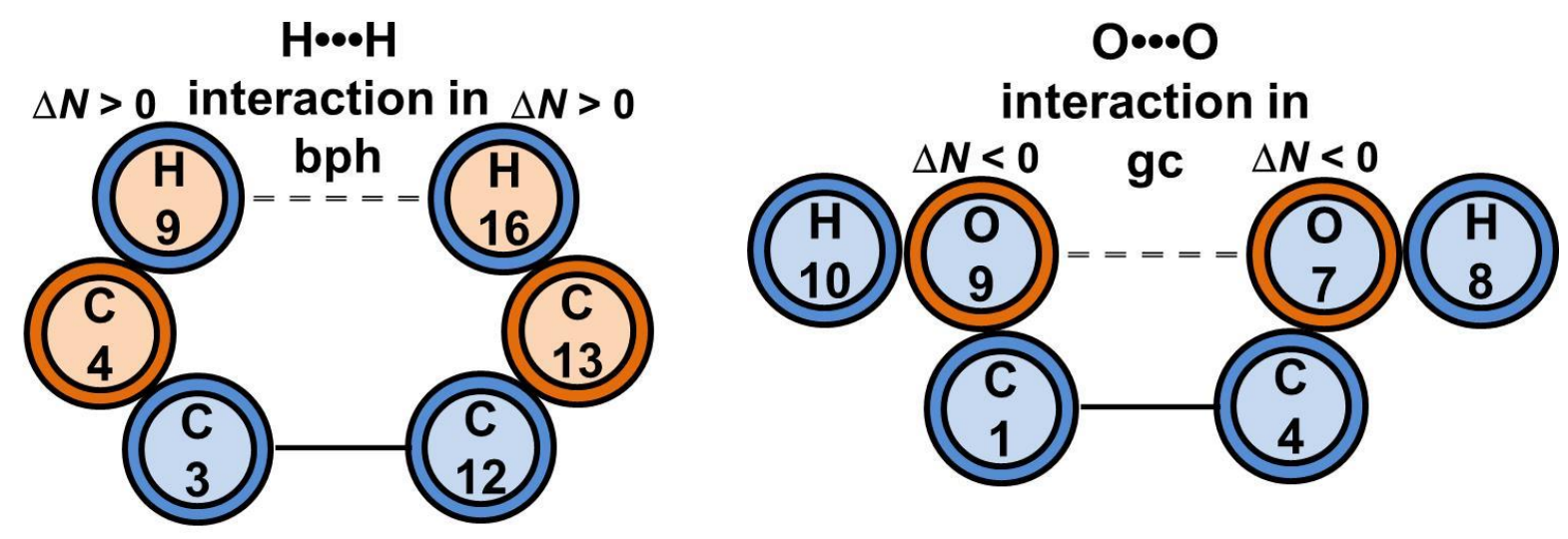

Fig. 4. A schematic representation of color-coded changes (blue $=$ decrease, brown $=$ increase) in atomic energies of indicated atoms in fin forms of $\mathbf{g c}$ and $\mathbf{b p h}$. Inner circle represents $\Delta E_{\text {self }}^{\mathrm{X}}$ (lighter color) and outer circle represents $\Delta E_{\text {add }}^{\mathrm{X}}$ (darker color). Negative (positive) values of $\Delta N$ indicate an outflow (inflow) of electrons on the interaction formation for atoms directly involved. A solid line stands for a covalent bond between $\mathrm{C}$-atoms. 
Moreover, opposite trends are observed for C-atoms to which either $\mathrm{H}$-atom (in bph) or O-atom (in gc) are bonded to: the $E_{\text {add }}^{\mathrm{C}}$ and $E_{\text {self }}^{\mathrm{C}}$ values of (i) $\mathrm{C} 4$ (and C13) in bph increased whereas (ii) $\mathrm{C} 1$ (and C4) in gc decreased. Also, an opposite trend in the electron population is observed for the $\mathrm{H}$-atoms involved in the steric hindrance in $\mathbf{b p h}$ when compared with clashing O-atoms of gc: a significant inflow, by $+11 \mathrm{~m} e$, is observed and these electrons are drawn mainly from the closest $\mathrm{C}$-atoms of the - $\mathrm{C} 5-\mathrm{C} 4-\mathrm{C} 3$ - fragment whereas large outflow takes place in the case of Oatoms of gc, as shown in Fig. 3.

Data presented in Table 2 and Figs. 3-4 also demonstrates that, as it was the case of Hen and Hea, there is no clear and easy for interpretation relationship between the change in the atoms' electron population and the $\Delta E_{\text {self }}^{\mathrm{X}}$ values. For instance, considering atoms involved in steric contacts: (i) a large outflow of electrons from O-atoms had a marginal effect on these atoms $E_{\text {self }}^{\mathrm{O}}$ but (ii) a large inflow of electrons to H-atoms in $\mathbf{b p h}$ is accompanied by a significant increase in $E_{\text {self }}^{\mathrm{H}}$. Furthermore, almost identical in value inflow of electrons is observed for all $\mathrm{H}$-atoms of the fin form of gc (see Fig. 3) but opposite trends in their self-atomic energy changes are observed; $\Delta E_{\text {self }}^{\mathrm{H} 10}=-1.9 \mathrm{kcal} \mathrm{mol}^{-1}$ whereas $\Delta E_{\text {self }}^{\mathrm{H}}=+0.5 \mathrm{kcal} \mathrm{mol}^{-1}$ is observed for $\mathrm{H} 2($ and H3).

Analysis of data shown in Table S8 in the SI reveals that changes in atomic properties found for $\mathrm{H} 9$ in bph (i) are very much different when compared with O-atoms in gc; exactly opposite variation is observed in five properties, namely, $\Delta E_{\mathrm{self}}^{\mathrm{X}}, \Delta T^{\mathrm{X}}, \Delta V_{\mathrm{ne}}^{\mathrm{XX}}, \Delta V_{\mathrm{ee}}^{\mathrm{XX}}$ and $\Delta N^{\mathrm{X}}$ (when an increase is observed for $\mathrm{O}$-atoms then a decrease is found in these properties for 'clashing' $\mathrm{H}$ atoms of bph, (ii) do not compare well at all with $\mathrm{H}$-atoms directly involved in the interactions in Hen and Hea as can be assessed from opposite trends in the $\Delta T^{\mathrm{X}}, \Delta V_{\mathrm{ne}}^{\mathrm{XX}}, \Delta V_{\mathrm{ee}}^{\mathrm{XX}}$ and $\Delta N^{\mathrm{X}}$ values, but most surprisingly (iii) almost replicate the trends found for heteroatoms in Hen and Hea; exactly the same variation is observed in six properties, namely, $\Delta E_{\text {self }}^{\mathrm{X}}, \Delta T^{\mathrm{X}}, \Delta V_{\mathrm{ne}}^{\mathrm{XX}}, \Delta V_{\mathrm{ee}}^{\mathrm{XX}}$ ,$\Delta N^{\mathrm{X}}$ and $\Delta \operatorname{Vol}^{\mathrm{X}}$. This strongly suggests that the physical nature of the $\mathrm{H} \cdot \bullet \cdot \mathrm{H}$ interaction in bph 
is unique among them all as it is neither similar to that found for steric $\mathrm{O}--\mathrm{O}$ contact in ge nor classical intramolecular interactions, $\mathrm{N} \bullet \bullet \mathrm{H}$ in Hen or $\mathrm{O} \bullet \bullet \cdot \mathrm{H}$ in Hea.

Analysis of data in Table 4 reveals that quite acceptable agreement is observed between B3LYP (SPC on MP2-optimized structures) and MP2 data obtained for C3, C4 and H9 (atoms of the bay). The only significant discrepancy was found for $\Delta E^{\mathrm{C} 4}\left(-0.3\right.$ and $0.1 \mathrm{kcal} \mathrm{mol}^{-1}$ at B3LYP and MP2, respectively), which indicates an opposite trend in this atom small energy change when going from ref to fin state of $\mathbf{b p h}$. Hence, it is safe to conclude that $\mathrm{C} 3$ of the link between the rings is most destabilized $\left(\Delta E^{\mathrm{X}}=21.6 \mathrm{kcal} \mathrm{mol}^{-1}\right.$ at MP2) for which largest (i) increase in atomic volume, (ii) decrease in atomic surface and (iii) decrease in $d_{\mathrm{e}}$ (average electron density in $\mathrm{Vol}^{\mathrm{X}}$ ) are observed (Table S8 in the SI).

\section{Table 4}

Relative to $r e f$ structure, changes in the QTAIM- and IQA-defined atomic energies for atoms in $\mathbf{b p h}$. $^{\text {a }}$

\begin{tabular}{lrrr}
\hline Atom X & $\Delta E_{\text {self }}^{\mathrm{X}}$ & \multicolumn{1}{c}{$\Delta T^{\mathrm{X}}$} & \multicolumn{1}{c}{$\Delta E^{\mathrm{X}}$} \\
\hline \multicolumn{2}{c}{ B3LYP (SPC on MP2 structures) } \\
\hline C3 & -3.9 & -20.6 & 21.0 \\
C4 & 1.2 & 0.6 & -0.3 \\
C5 & 0.5 & 1.2 & -1.0 \\
C6 & 0.3 & 3.1 & -2.8 \\
H9 & 1.7 & 6.9 & -7.0 \\
H10 & 0.0 & 0.5 & -0.5 \\
H1 & 0.0 & -0.4 & 0.4 \\
---5 & MP2 & & \\
- C3 & -3.5 & -20.6 & 21.6 \\
C4 & 0.9 & 0.8 & 0.1 \\
H9 & 1.7 & 7.8 & -7.8 \\
\hline
\end{tabular}

${ }^{a}$ Energies are in kcal mol ${ }^{-1}$. For more details, see a footnote of Table 3. Due to molecular symmetry, only data for representative atoms are included.

Moreover, it appears that ortho-hydrogens are most stabilized $\left(\Delta E^{\mathrm{X}}=-7.8 \mathrm{kcal} \mathrm{mol}^{-1}\right.$ at MP2) and characterized by the largest decrease in atomic volume and increase in $d_{\mathrm{e}}$. Notice that significant changes in atomic properties are also observed for other C-atoms of the ring in $\mathbf{b p h}$. 
To conclude this section we would like to stress that the largest changes in the additive and self-atomic energies are indeed observed for atoms involved in the classical intramolecular stabilizing interactions in the fin forms of Hen and Hea. In case of gc, $E_{\text {add }}^{\mathrm{O}}$ and $E_{\text {self }}^{\mathrm{C}}$ changed the most. Considering bph, order(s) of magnitude smaller changes in atomic energies are observed altogether. Furthermore, when the total change in the self-atomic energies of all atoms in molecules investigated here is considered, we found $\Delta \sum_{\mathrm{X}} E_{\text {self }}^{\mathrm{X}}>0$ for Hen and Hea but this energy term decreased for $\mathbf{g c}$ where steric hindrance is present. However, the trend found for $\mathbf{g c}$ is not reproduced in bph and the $\sum_{\mathrm{X}} E_{\mathrm{self}}^{\mathrm{X}}$ energy term increased by about $6 \mathrm{kcal} \mathrm{mol}^{-1}$ in the planar bph at the B3LYP level.

\subsection{Exploring the changes in the diatomic interaction energies}

We will explore now the second fundamental term in the IQA-based partitioning scheme, diatomic interaction energies. It is important and informative, however, to learn first about the interaction energies between atoms of the selected $\mathscr{G}$ fragments in the ref structures of molecules investigated here (see Fig. S3 in the SI). We obtained the $E_{\mathrm{int}}^{\mathrm{AB}}$ values of -40.3 for $\{\mathrm{N} 7, \mathrm{H} 11\},-$ 45.0 for $\{\mathrm{O} 7, \mathrm{H} 12\}, 111.2$ for $\{\mathrm{O} 7, \mathrm{O} 9\}$, and $-0.8 \mathrm{kcal} \mathrm{mol}^{-1}$ for $\{\mathrm{H} 8, \mathrm{H} 16\}$ in ref structures of Hen, Hea, gc and bph, respectively, where corresponding interatomic distances d(A,B) were found to be 4.088, 3.955, 3.601 and $2.532 \AA$. Clearly, even though atoms A and B in the linear conformers of Hen, Hea and gc cannot be seen as nearest neighbors (they are separated by two $\mathrm{CH}_{2}$ - fragments) they strongly interact with each other. This observation supports the approach taken here where variation in the diatomic interaction energies for all pairs of atoms, $\Delta E_{\mathrm{int}}^{\mathrm{XY}}$, is accounted for without making any simplifying assumption. From that also follows that $\sum_{\mathrm{X} \neq \mathrm{A}} 0.5 E_{\mathrm{int}}^{\mathrm{AX}}$ (recall that this contributes to the additive atomic energy of a given atom) does not necessarily represent a truly localized energy term of an extensive system. 


\subsubsection{Interactions in Hen and Hea}

Analysis of data shown in Tables S9-S12 in the SI (for convenience, selected strongest stabilizing and destabilizing in nature changes in diatomic interactions are shown in Table 5) leads to the following observations:

- By far the largest $\Delta E_{\mathrm{int}}^{\mathrm{XY}}$ values are observed for atoms of the $\mathscr{L}_{\mathscr{L}}$ fragments; a change in a single interaction, $\Delta E_{\mathrm{int}}^{\mathrm{XY}} \approx-55 \mathrm{kcal} \mathrm{mol}^{-1}$ for $\mathrm{N} 7 \cdot \bullet \cdot \mathrm{H} 11$ and $\Delta E_{\mathrm{int}}^{\mathrm{XY}} \approx-38 \mathrm{kcal} \mathrm{mol}^{-1}$ for $\mathrm{O} 7 \cdot \bullet \cdot \mathrm{H} 12$, constitutes about 130 and $300 \%$ of the total change in all interaction energies in Hen and Hea, respectively.

- Moreover, the N7 and O7 atoms of the G fragments in Hen and Hea, respectively, are also involved in other most stabilizing contributions, e.g., $\Delta E_{\mathrm{int}}^{\mathrm{N} 7 \mathrm{H} 13}=-14.0 \mathrm{kcal} \mathrm{mol}^{-1}$ in $\mathbf{H e n}$ and $\Delta E_{\mathrm{int}}^{\mathrm{O} \mathrm{H} 11}=-12.5 \mathrm{kcal} \mathrm{mol}^{-1}$ in Hea.

- All most significant $\Delta E_{\mathrm{int}}^{\mathrm{XY}}$ values are dominated by the classical term, $\Delta V_{\mathrm{cl}}^{\mathrm{XY}}$.

\section{Table 5}

Most significant, stabilizing and destabilizing, contributions coming from diatomic interaction energy and its components in Hen and Hea. ${ }^{a}$

\begin{tabular}{|c|c|c|c|c|c|c|c|c|c|}
\hline \multicolumn{2}{|c|}{ atoms } & \multirow{2}{*}{$\Delta E_{\mathrm{int}}^{\mathrm{XY}}$} & \multirow{2}{*}{$\Delta V_{\mathrm{cl}}^{\mathrm{XY}}$} & \multirow{2}{*}{$\Delta V_{\mathrm{XC}}^{\mathrm{XY}}$} & \multicolumn{2}{|c|}{ atoms } & \multirow{2}{*}{$\Delta E_{\mathrm{int}}^{\mathrm{XY}}$} & \multirow{2}{*}{$\Delta V_{\mathrm{cl}}^{\mathrm{XY}}$} & \multirow{2}{*}{$\Delta V_{\mathrm{XC}}^{\mathrm{XY}}$} \\
\hline $\mathrm{X}$ & $\mathrm{Y}$ & & & & $X$ & $\mathrm{Y}$ & & & \\
\hline \multicolumn{10}{|c|}{ Hen } \\
\hline N7 & H11 & -54.7 & -45.2 & -9.5 & $\mathrm{H} 8$ & H11 & 9.4 & 9.5 & -0.1 \\
\hline $\mathrm{C} 4$ & N7 & -15.0 & -15.5 & 0.5 & H9 & H11 & 10.7 & 10.8 & -0.1 \\
\hline N7 & H13 & -14.0 & -13.9 & -0.1 & N7 & N10 & 41.2 & 46.8 & -5.6 \\
\hline \multicolumn{10}{|c|}{ Hea } \\
\hline O7 & H12 & -37.8 & -33.8 & -4.0 & $\mathrm{C} 1$ & H12 & 10.0 & 10.0 & 0.1 \\
\hline H8 & N9 & -12.8 & -12.8 & 0.0 & H8 & H12 & 11.9 & 12.0 & 0.0 \\
\hline O7 & H11 & -12.5 & -12.5 & 0.0 & 07 & N9 & 31.9 & 36.2 & -4.3 \\
\hline
\end{tabular}

- A large spread in the stabilizing and destabilizing contributions is observed, but the total change in all diatomic interaction energies (the $\Delta 0.5 \sum_{\mathrm{X}} \sum_{\mathrm{Y} \neq \mathrm{X}} E_{\mathrm{int}}^{\mathrm{XY}}$ energy term, $-41.2(\mathbf{H e n})$ and - 
12.5 (Hea) $\mathrm{kcal} \mathrm{mol}^{-1}$ ) significantly contributes to the stability of the fin structures and it follows the trend in $\Delta E$ values of these molecules.

- Finally, note that some interactions changed in unfavorable manner and the largest contributions involve heteroatoms, $\Delta E_{\mathrm{int}}^{\mathrm{N} 7 \mathrm{~N} 10}=41.2 \mathrm{kcal} \mathrm{mol}^{-1}$ in Hen and $\Delta E_{\mathrm{int}}^{\mathrm{O} \mathrm{N} 9}=31.9 \mathrm{kcal}$ $\mathrm{mol}^{-1}$ in Hea. Although smaller in value, they are significant and comparable with contributions made by the leading stabilizing N7 $\bullet \bullet H 11$ in Hen and O7 $\bullet \bullet H 12$ in Hea. This illustrates that the formation of an intramolecular bonding interaction is a complex and multi-atomic 'affair', hence to understand the origin of observed $\Delta E$ when a molecule changes from ref to fin state, all these changes must be accounted for.

\subsubsection{Analysis of interactions in $\mathrm{gc}$}

Data shown in Table 6 (see also Table S11 in the SI where a full set of data is included) reveals that by far much larger spread in values, when compared with Hen and Hea, is observed in gc. Somewhat surprisingly, the largest change does not involve the O7--O9 contact but rather these atoms interactions with $\mathrm{C}$-atoms. We found $\Delta E_{\mathrm{int}}^{\mathrm{XY}}$ of $-283.2 \mathrm{kcal} \mathrm{mol}^{-1}$ for covalently bonded $\mathrm{C} 1$ and $\mathrm{O} 9$ atoms and $+279.5 \mathrm{kcal} \mathrm{mol}^{-1}$ for the non-bonded $\mathrm{C} 4$ and $\mathrm{O} 9$ atoms; both components, $\Delta V_{\mathrm{cl}}^{\mathrm{XY}}$ and $\Delta V_{\mathrm{XC}}^{\mathrm{XY}}$, changed a great deal but the latter considerably more. Another and unexpected finding is related to the non-bonded interactions between $\mathrm{H}$-atoms of the functional $-\mathrm{OH}$ groups and C-atoms. We found $\Delta E_{\mathrm{int}}^{\mathrm{H} 10 \mathrm{C} 1}=+43.9 \mathrm{kcal} \mathrm{mol}^{-1}$ involving the Catom to which the $-\mathrm{OH} 10$ group is bonded to and $\Delta E_{\mathrm{int}}^{\mathrm{H} 10 \mathrm{C} 4}=-41.4 \mathrm{kcal} \mathrm{mol}^{-1}$ involving a distant C-atom. As one would expect, these interactions are entirely of classical nature and much unexpectedly over 50\% larger in value than that found for the O-atoms involved in the steric clash. Due to the molecular symmetry, the transformation from the staggered to eclipsed form of gc generated a number of paired, opposite in nature but similar in value interactions. Because of that, these large changes cancelled each other almost completely. 


\section{Table 6}

Most significant, stabilizing and destabilizing, contributions coming from diatomic interaction energy and its components in $\mathbf{g c}$ and $\mathbf{b p h} \mathbf{~}^{\mathrm{a}}$

\begin{tabular}{|c|c|c|c|c|c|c|c|c|c|}
\hline \multicolumn{2}{|c|}{ atoms } & \multirow{2}{*}{$\Delta E_{\mathrm{int}}^{\mathrm{XY}}$} & \multirow{2}{*}{$\Delta V_{\mathrm{cl}}^{\mathrm{XY}}$} & \multirow{2}{*}{$\Delta V_{\mathrm{XC}}^{\mathrm{XY}}$} & \multicolumn{2}{|c|}{ atoms } & \multirow{2}{*}{$\Delta E_{\mathrm{int}}^{\mathrm{XY}}$} & \multirow{2}{*}{$\Delta V_{\mathrm{cl}}^{\mathrm{XY}}$} & \multirow{2}{*}{$\Delta V_{\mathrm{XC}}^{\mathrm{XY}}$} \\
\hline$X$ & $\mathrm{Y}$ & & & & $X$ & $\mathrm{Y}$ & & & \\
\hline \multicolumn{10}{|c|}{ gc } \\
\hline $\mathrm{C} 1$ & O9 & -283.2 & -132.7 & -150.6 & 07 & O9 & 25.8 & 34.0 & -8.2 \\
\hline $\mathrm{C} 4$ & H10 & -41.4 & -42.3 & 0.8 & $\mathrm{C} 1$ & H10 & 43.9 & 44.8 & -0.9 \\
\hline 07 & H10 & -9.9 & -9.8 & -0.1 & $\mathrm{C} 4$ & O9 & 279.5 & 126.8 & 152.8 \\
\hline \multicolumn{10}{|c|}{ bph } \\
\hline H9 & H16 & -3.8 & 0.0 & -3.8 & C3 & $\mathrm{C} 4$ & 0.7 & -0.2 & 1.0 \\
\hline $\mathrm{C} 4$ & H16 & -0.2 & 0.0 & -0.3 & $\mathrm{C} 12$ & $\mathrm{C} 13$ & 0.7 & -0.2 & 1.0 \\
\hline H9 & $\mathrm{C} 13$ & -0.2 & 0.0 & -0.3 & $\mathrm{C} 3$ & $\mathrm{C} 12$ & 1.1 & -0.1 & 1.2 \\
\hline
\end{tabular}

${ }^{\mathrm{a}}$ All values in $\mathrm{kcal} \mathrm{mol}^{-1}$ at MP2.

Focusing on $\Delta E_{\mathrm{int}}^{\mathrm{0709}}$, we found that this contribution is of strong repulsive nature, $26 \mathrm{kcal}$ $\mathrm{mol}^{-1}$, and dominated by a classical term. Even though it is only third in value amongst the repulsive interactions and an order of magnitude smaller when compared with these atoms interactions with $\mathrm{C}$-atoms, it is the leading interaction which counteracts the unfavorable change from the ref (staggered) to the fin (eclipsed) form. Notice that the change in all diatomic interaction energies, and this includes also $\Delta E_{\mathrm{int}}^{\mathrm{O} 09}$ of $+26 \mathrm{kcal} \mathrm{mol}^{-1}$, was found to be $+17.2 \mathrm{kcal}$ $\mathrm{mol}^{-1}$ and this clearly shows that all the other contributions, even though individually large, became less significant due to the paired interaction effect discussed above.

\subsubsection{Analysis of interactions in $\mathbf{b p h}$}

To gain an insight on the significance of MP2 values obtained for bph (see Table 6 and Table S12 in the SI) it was imperative to analyze a full set of data originated from a wavefunction obtained from a SPC at the B3LYP level performed on the MP2 optimized structures. To asses reliability of B3LYP data we compared the sums of halved interaction energies, $\sum_{\mathrm{X} \neq \mathrm{A}} 0.5 E_{\mathrm{int}}^{\mathrm{AX}}$, because this energy term (i) involves contributions coming from interactions with all atoms in a molecule and (ii) is always generated for any atom selected for the IQA calculations, hence it 
was also available at the MP2 level. Relevant data obtained for representative atoms of the bay at both levels of theory are presented in Table 7 (a full B3LYP data set is included in Table S13 in the SI).

First, one must note that the changes in this energy term obtained for atoms of interest agree qualitatively well and the observed at the MP2 level trends for different atoms are reproduced at the B3LYP level (the only exemption is the small XC-term for $\mathrm{C} 4$ which was found to be 0.1 and $-0.1 \mathrm{kcal} \mathrm{mol}^{-1}$ at the MP2 and B3LYP level, respectively). Importantly, data shown in Table 7 clearly indicates that ortho-H atoms experience more favorable interactions with remaining atoms in the planar form of $\mathbf{b p h},-1.9$ (MP2) and $-1.8 \mathrm{kcal} \mathrm{mol}^{-1}$ (B3LYP) whereas exactly opposite applies to C-atoms of the link, +2.8 (MP2) and +2.2 (B3LYP).

\section{Table 7}

Comparison of indicated energy terms obtained for selected atoms of bph at the MP2 and B3LYP levels of theory.

\begin{tabular}{|c|c|c|c|c|c|}
\hline \multirow[t]{2}{*}{ Atom A } & \multicolumn{2}{|c|}{$\sum_{\mathrm{X} \neq \mathrm{A}} 0.5 E_{\mathrm{int}}^{\mathrm{AX} \mathrm{a}}$} & \multirow{2}{*}{$\Delta \sum_{\mathrm{X} \neq \mathrm{A}} 0.5 E_{\mathrm{int}}^{\mathrm{AX} \mathrm{b}}$} & \multirow{2}{*}{$\Delta \sum_{\mathrm{X} \neq \mathrm{A}} 0.5 V_{\mathrm{cl}}^{\mathrm{AX} \mathrm{b}}$} & \multirow{2}{*}{$\Delta \sum_{\mathrm{X} \neq \mathrm{A}} 0.5 V_{\mathrm{XC}}^{\mathrm{AX} b}$} \\
\hline & ref & fin & & & \\
\hline & & & MP2 & & \\
\hline H9 & -0.1183 & -0.1214 & -1.9 & -0.1 & -1.8 \\
\hline $\mathrm{C} 4$ & -0.4543 & -0.4540 & 0.2 & 0.1 & 0.1 \\
\hline \multirow[t]{2}{*}{ C3 } & -0.4775 & -0.4730 & 2.8 & -0.3 & 3.1 \\
\hline & & & B3LY & & \\
\hline H9 & -0.1288 & -0.1317 & -1.8 & -0.2 & -1.6 \\
\hline $\mathrm{C} 4$ & -0.5040 & -0.5038 & 0.1 & 0.1 & -0.1 \\
\hline $\mathrm{C} 3$ & -0.5288 & -0.5255 & 2.2 & -0.2 & 2.4 \\
\hline
\end{tabular}

Acceptable consistency in the available MP2 and B3LYP data provided necessary confidence in the analysis of trends found at the B3LYP level (Table S13 in the SI). Overall, diatomic interaction energies changed very little, when compared with other molecules investigated in this work. Furthermore, from the analysis of 231 values of $\Delta E_{\text {int }}^{\mathrm{XY}}$ in $\mathbf{b p h}$ it follows that the one obtained for the ortho-H atoms (i) is most significant among all, by about $2 \mathrm{kcal} \mathrm{mol}^{-1}$ in 
absolute value, (ii) contributes in a stabilizing manner as we obtained $\Delta E_{\mathrm{int}}^{\mathrm{H} 9 \mathrm{H} 16}$ of $-3.3 \mathrm{kcal} \mathrm{mol}^{-1}$ $\left(-3.8 \mathrm{kcal} \mathrm{mol}^{-1}\right.$ at the MP2) and (iii) is entirely of the exchange-correlation origin $\left(\Delta V_{\mathrm{cl}}^{\mathrm{H} 9 \mathrm{H} 16}=\right.$ $0.003 \mathrm{kcal} \mathrm{mol}^{-1}$ was found at MP2). We have also established (see Table S13 in the SI) that (i) 'clashing' H-atoms are characterized by most significant, by about $1.5 \mathrm{kcal} \mathrm{mol}^{-1}$, stabilizing contribution made by these atoms interactions with remaining atoms of $\mathbf{b p h}$, and (ii) the same energy term obtained for the $\mathrm{C}$-atoms of the link is the largest (by about $1.9 \mathrm{kcal} \mathrm{mol}^{-1}$ ) among all destabilizing contributions in the planar bph.

Finally, note that the $\mathrm{H} 9 \cdot \bullet \mathrm{H} 16$ interaction is stabilizing in $r e f\left(-0.8 \mathrm{kcal} \mathrm{mol}^{-1}\right)$ and fin $(-3.8$ $\mathrm{kcal} \mathrm{mol}^{-1}$ ) forms of $\mathbf{b p h}$ and significantly stronger in the planar, higher energy conformer. Hence, it strongly suggests that this interaction does not drive a structural change from the planar to the lower energy conformer of $\mathbf{b p h}$.

\subsection{FAMSEC-based interpretation of intramolecular interactions}

\subsubsection{Interpretation of intramolecular interactions (bonds) in $\mathbf{H e n}$ and $\mathbf{H e a}$}

Firstly note that the same trends in all specified energy components are observed in both molecules even though a BP is not present in Hea (see Table 8). Furthermore, the energy contributions, $E_{\text {attr-mol }}^{\mathscr{E}}$ of $-10.8\left(\right.$ Hen) and $-2.3 \mathrm{kcal} \mathrm{mol}^{-1}$ (Hea), which are attributed to the $\mathscr{G}$ fragments add to the overall stability of the respective molecules with $\Delta E_{\mathrm{int}}^{\mathrm{AB}}$ of -54.7 (for Hen) and $-37.8 \mathrm{kcal} \mathrm{mol}^{-1}$ (for Hea) being the largest among the contributing components. Although the mol-FAMSEC values follow the general trend in $\Delta E$, this term is over twice as large when compared with $\Delta E$ of Hen and about $30 \%$ smaller then $\Delta E$ in the case of Hea. This clearly

illustrates that the computed $\Delta E$ should not be attributed solely to the formation of the intramolecular interactions but is the overall result of energy-contributing changes taking place throughout a molecule when it changes from the ref to fin state. 


\section{Table 8}

Energy components used for the interpretation of intramolecular interactions in molecules investigated in this work. ${ }^{\mathrm{a}}$

\begin{tabular}{|c|c|c|c|c|}
\hline energy term & $\begin{array}{c}\text { Hen } \\
\{\mathbf{N 7 , H 1 1}\}\end{array}$ & $\begin{array}{c}\text { Hea } \\
\{07, \text { H12 }\} \\
\end{array}$ & $\begin{array}{c}\text { bph } \\
\{\text { H9,H16 }\}\end{array}$ & $\begin{array}{c}\text { gc } \\
\{07,09\}\end{array}$ \\
\hline$E_{\text {attr-mol }}^{\mathscr{E}}$ & -10.8 & -2.3 & -0.7 & 13.1 \\
\hline$\Delta E_{\mathrm{int}}^{\mathrm{AB}}$ & -54.7 & -37.8 & -3.8 & 25.8 \\
\hline$\Delta \sum_{\mathrm{X} \neq \mathrm{A}} E_{\mathrm{int}}^{\mathrm{AX}}$ & $\begin{array}{r}-65.0 \\
(A=N 7)\end{array}$ & $\begin{array}{r}-36.8 \\
(\mathrm{~A}=\mathrm{O} 7)\end{array}$ & -3.9 & 19.7 \\
\hline & $\begin{array}{r}-22.4 \\
(\mathrm{~A}=\mathrm{H} 11)\end{array}$ & $\begin{array}{r}-13.0 \\
(\mathrm{~A}=\mathrm{H} 12)\end{array}$ & - & - \\
\hline$\Delta E_{\mathrm{int}}^{\mathrm{A} \mathscr{F}}$ & $\begin{array}{r}-10.3 \\
(\mathrm{~A}=\mathrm{N} 7)\end{array}$ & $\begin{array}{r}1.0 \\
(\mathrm{~A}=\mathrm{O} 7)\end{array}$ & -0.1 & -8.6 \\
\hline & $\begin{array}{r}32.3 \\
(\mathrm{~A}=\mathrm{H} 11)\end{array}$ & $\begin{array}{r}24.8 \\
(\mathrm{~A}=\mathrm{H} 12)\end{array}$ & - & - \\
\hline$\Delta E_{\mathrm{int}}^{\mathscr{E} \mathcal{F}}$ & 22.1 & 25.8 & -0.2 & -12.2 \\
\hline$\Delta 0.5 \sum_{\mathrm{X}} \sum_{\mathrm{Y} \neq \mathrm{X}} E_{\mathrm{int}}^{\mathrm{X}, \mathrm{Y}}$ & -41.2 & -12.6 & - & 17.2 \\
\hline$\Delta 0.5 \sum_{\mathrm{X}} \sum_{\mathrm{Y} \neq \mathrm{A}, \mathrm{B}} E_{\mathrm{int}}^{\mathrm{X}, \mathrm{Y}}$ & 13.5 & 25.2 & - & -8.7 \\
\hline$\Delta E_{\text {self }}^{\mathscr{G}}$ & 21.9 & 9.8 & 3.3 & -0.5 \\
\hline$\Delta \sum_{\mathrm{X}} E_{\mathrm{self}}^{\mathrm{X}}$ & 26.2 & 3.2 & - & -7.2 \\
\hline$\Delta E_{\text {add }}^{\mathscr{G}}$ & -21.8 & -15.2 & -0.6 & 19.2 \\
\hline$E_{\text {attr-loc }}^{\mathscr{E}}$ & -32.9 & -28.0 & -0.5 & 25.3 \\
\hline
\end{tabular}

Focusing on individual atoms of the $\mathscr{E}$ fragments, it appears that they found themselves in overall more favorable interatomic environment in the fin forms of the molecules because $\Delta \sum_{\mathrm{X} \neq \mathrm{A}} E_{\mathrm{int}}^{\mathrm{AX}}<0$ (the sum of diatomic interaction energies with remaining atoms). This is particularly true for N7 in Hen, the only atom of the Gूgagments for which the interactions with remaining atoms (atoms of the $\mathscr{\Psi}$ fragment) also contributed in a stabilizing manner, $\Delta E_{\mathrm{int}}^{\mathrm{N} 7 \mathscr{F}}=-$ $10.3 \mathrm{kcal} \mathrm{mol}^{-1}$. Interestingly, the $\mathrm{H}$-atoms the $\mathcal{G}_{\text {fragments in }}$ both molecules experience most unfavorable interactions with atoms of the molecular fragment $\mathscr{H}$; we found $\Delta E_{\mathrm{int}}^{\mathrm{A} \mathscr{F}}$ of 32.3 and $24.8 \mathrm{kcal} \mathrm{mol}^{-1}$ for $\mathrm{H} 11$ and $\mathrm{H} 12$ in Hen and Hea, respectively. Also their interfragment 
interaction energies $\Delta E_{\text {int }}^{\text {EF }}$ are unfavorable $\left(22.1\right.$ and $25.8 \mathrm{kcal} \mathrm{mol}^{-1}$ ) but about 2.5 and 1.5 times less significant than the relevant $\Delta E_{\mathrm{int}}^{\mathrm{AB}}$ values of leading single diatomic interactions in Hen and Hea, respectively.

By definition, the mol-FAMSEC term accounts only for the interactions involving atoms of the $\mathcal{G}$ fragment and there are 23 out of 78 and 21 out 66 such interactions in Hen and Hea, respectively. The total change in the 78 and 66 interaction energies in Hen and Hea (this includes $\Delta E_{\mathrm{int}}^{\mathrm{AB}}$ ) was found to be -41.2 and $-12.6 \mathrm{kcal} \mathrm{mol}^{-1}$, respectively. This means that the leading interactions constitute about $133(\mathrm{~N} 7 \cdot \bullet \cdot \mathrm{H} 11$ in Hen) and $300(\mathrm{O} 7 \bullet \bullet \bullet H 12$ in Hea) \% of the summed energy effect of all interactions in a molecule. Furthermore, the combined terms, $\Delta E_{\mathrm{int}}^{\mathrm{AB}}+\Delta E_{\mathrm{int}}^{\mathscr{E} \mathscr{F}}$ (this represents all possible diatomic interactions atoms of the $\mathscr{G}$ fragment are involved in) contribute -32.7 and $-12.1 \mathrm{kcal} \mathrm{mol}^{-1}$ which constitutes 79 and $95 \%$ of the total change in diatomic interaction energies, in Hen and Hea, respectively. Perhaps even more informative is comparison of contributions made by the $\mathrm{A} \bullet \bullet \mathrm{B}$ interaction against energy contribution made by all remaining interactions in a molecule; for the latter we obtained 13.5 and $25.2 \mathrm{kcal} \mathrm{mol}^{-1}$ for Hen and Hea, respectively. This exemplifies significance of the leading interactions in these molecules as they contribute 4 and 1.5 times more to stability of fin forms of Hen and Hea, respectively, than the overall destabilizing contribution coming from all other interactions.

As expected and also shown in section 4.1.1, the self-atomic energies of atoms involved in the interactions increased; we found $\Delta E_{\text {self }}^{\complement}$ of 21.9 and $9.8 \mathrm{kcal} \mathrm{mol}^{-1}$ for Hen and Hea, respectively. Hence, there are two sources of destabilizing contributions $\left(\Delta E_{\mathrm{self}}^{\mathscr{E}}\right.$ and $\left.\Delta E_{\mathrm{int}}^{\mathfrak{E} \mathscr{F}}\right)$ and, when combined, one obtains 44.0 and $35.6 \mathrm{kcal} \mathrm{mol}^{-1}$ for Hen and Hea, respectively. These values, however, are compensated over by $\Delta E_{\mathrm{int}}^{\mathrm{AB}}$ and this is why the mol-FAMSEC term correlates well with a general trend observed for $\Delta E$ for these two molecules. Another important aspect is to assess relative significance of contributions made by total changes in self-atomic and interaction 
energies. We found $\Delta \sum_{\mathrm{X}} E_{\mathrm{self}}^{\mathrm{X}}$ of 26.2 and $3.2 \mathrm{kcal} \mathrm{mol}^{-1}$ which is significantly smaller (by 15 and $9 \mathrm{kcal} \mathrm{mol}^{-1}$ ) for Hen and Hea, respectively, when compared with the contributions made by all interactions.

The atoms of the $\mathscr{G}$ fragments in Hen and Hea are characterized by $\Delta E_{\text {add }}^{\mathscr{E}}<0$ and this can be easily explained by favorable energy contribution coming from the interactions with all the remaining atoms, $\left|\Delta \sum_{\mathrm{X} \neq \mathrm{A}} 0.5 E_{\mathrm{int}}^{\mathrm{A}, \mathrm{X}}\right|>\Delta E_{\text {self }}^{\mathrm{A}}$. As a result, $\Delta E_{\text {add }}^{\mathscr{E}}$ is highly favorable in both molecules, -21.8 (Hen) and -15.2 (Hea) $\mathrm{kcal} \mathrm{mol}^{-1}$. Computing of all unique diatomic interactions in ref and fin makes it possible to generate a kind of topology of this energy change. This information is invaluable also in tracing the origin of trends observed in additive atomic energies (Table 2). Focusing just on heteroatoms in Hen and Hea, the observed decrease in $E_{\text {add }}^{\mathrm{N} 7}$ (in Hen) and $E_{\text {add }}^{\mathrm{O} 7}$ (in Hea) can be attributed mainly to stabilizing interactions with H-atoms of the $-\mathrm{NH}_{3}{ }^{+}$functional groups.

Finally, $E_{\text {attr-loc }}^{\mathscr{E}}$ can be estimated by combining changes in self-atomic energies, $\Delta E_{\text {self }}^{\mathscr{E}}$, and the interaction energy between atoms involved, $\Delta E_{\mathrm{int}}^{\mathrm{AB}}$; in both cases these molecules' $\mathscr{Z}_{\mathrm{g}}$ fragments are highly stabilized, by about $-30 \mathrm{kcal} \mathrm{mol}^{-1}$.

All the above demonstrates that the computed FAMSEC terms, correlate well with a classical notion of the stabilizing nature of the $\mathrm{NH} \bullet \cdot \mathrm{N}$ and $\mathrm{NH} \cdots \cdot \mathrm{O}$ intramolecular interactions. Furthermore, the stabilizing energy contributions made by $E_{\text {attr-loc }}^{\mathscr{E}}$ (confined to a molecular fragment $\left.\mathscr{G}^{\prime}\right), \Delta E_{\mathrm{int}}^{\mathrm{AB}}\left(\right.$ diatomic $\mathrm{A} \bullet \bullet \mathrm{B}$ interaction) and $\Delta \sum_{\mathrm{X} \neq \mathrm{A}} E_{\mathrm{int}}^{\mathrm{A}, \mathrm{X}}$ (interactions of atom $\mathrm{A}$ with all remaining atoms in a molecule) correlate with a classical notion that the atoms of $Z_{\text {are }}$ not strained on getting spontaneously involved in the stabilizing intramolecular interaction, which is further supported by the $\Delta E_{\text {add }}^{\mathscr{G}}$ term. 


\subsubsection{Interpretation of the steric clashes in $\mathrm{gc}$ and $\mathbf{b p h}$}

It was imperative to establish first whether FAMSEC-based analysis can differentiate a classical steric clash in gc from H-bonding type of interactions and we used the same set of energy terms as discussed above for Hen and Hea. A quick inspection of data shown in Table 8 immediately reveals that the physical nature of the $\mathrm{O} \bullet \bullet \mathrm{O}$ interaction in $\mathbf{g c}$ is very different when compared with those obtained for Hen and Hea. Both FAMSEC terms, $E_{\text {attr-mol }}^{\mathscr{E}}$ and $E_{\text {attr-loc }}^{\mathscr{E}}$, are large and positive due to highly repulsive interaction O-atoms are involved in, $\Delta E_{\text {int }}^{\mathrm{OO}}=25.8 \mathrm{kcal}$ $\mathrm{mol}^{-1}$. Furthermore, the energy terms, $\Delta \sum_{\mathrm{X} \neq \mathrm{A}} E_{\mathrm{int}}^{\mathrm{AX}}, \Delta 0.5 \sum_{\mathrm{X}} \sum_{\mathrm{Y} \neq \mathrm{X}} E_{\mathrm{int}}^{\mathrm{X}, \mathrm{Y}}, \Delta E_{\text {add }}^{\mathcal{E}}$ and $E_{\mathrm{attr}-\text { loc }}^{\mathscr{E}}$, are also large and positive (hence a net energy of the G্G fragment has increased) which is exactly opposite to what we found for a spontaneously formed stabilizing intramolecular interaction in Hen and Hea. Interestingly and importantly, the highly repulsive and forced-to-be interaction between Oatoms can be seen as predominantly, if not entirely, responsible for the increase in energy of the fin form of gc. Note that, when $\Delta E_{\mathrm{int}}^{\mathrm{OO}}$ is excluded, then (i) interactions of each individual Oatom with remaining atoms $\left(-8.6 \mathrm{kcal} \mathrm{mol}^{-1}\right)$ (ii) interactions of the $\mathscr{G}=\{\mathrm{O}, \mathrm{O}\}$ fragment with atoms of the fragment $\mathscr{\Psi}\left(-12.2 \mathrm{kcal} \mathrm{mol}^{-1}\right)$ as well as (iii) the total change in all remaining interactions in the fin form of $\mathbf{g c}\left(-8.7 \mathrm{kcal} \mathrm{mol}^{-1}\right)$ are all favorable.

An increase in $E_{\text {self }}^{\mathrm{X}}$ can be seen as a pre-organization energy or an energy penalty an atom must pay on a bonding interaction formation and all atoms directly involved in intramolecular interactions in Hen and Hea do indeed are characterized by larger $E_{\text {self }}^{\mathrm{x}}$ values relative to the ref state of these molecules. Just opposite is observed for O-atoms in eclipsed gc which strongly supports a general notion that they are not involved in a bonding interaction. Also, the additive atomic energies of O-atoms are highly positive (they are destabilized).

In general, data shown in Table 8 fully recovers (i) the repulsive nature of the O--O steric clash and its destabilizing energy contributions made to entire molecule as well as to the molecular fragment $\mathscr{G}$ which fully supports a classical notion of steric hindrance. Finally, the 
O7••09 interaction might be seen as driving a molecule to the lower energy state and this is the only important similarity between this interaction and those in Hen and Hea.

Having established that the proposed methodology was able to fully distinguish between classical stabilizing intramolecular interactions and steric clash, gave us confidence needed to interpret the steric H9--H16 contact in bph. It became instantly obvious, from the analysis of data in Table 8, that its physical nature is very different than that uncovered for the $\mathrm{O} 7--\mathrm{O} 9$ clash and that ortho-H do not conform to the classical notion of strained atoms involved in steric clash. Also, it is apparent that the $\mathrm{H} 9 \cdot \bullet \mathrm{H} 16$ interaction is not responsible for the increase in $E$ of the planar form of bph because this molecular $\mathscr{G}=\{\mathrm{H} 9, \mathrm{H} 16\}$ fragment contributes (i) to the entire molecule $\left(E_{\text {attr-mol }}^{\mathrm{H} 9 \mathrm{H} 16}=-0.7 \mathrm{kcal} \mathrm{mol}^{-1}\right)$ and locally $\left(E_{\text {attr-loc }}^{\mathcal{E}}=-0.5 \mathrm{kcal} \mathrm{mol}^{-1}\right)$ in a stabilizing manner as well as its interactions with atoms of $\mathscr{H}$, the interfragment energy component $\left(\Delta E_{\mathrm{int}}^{\mathrm{A} \mathscr{H}}\right.$ $=-0.2 \mathrm{kcal} \mathrm{mol}^{-1}$ ) is stabilizing. Furthermore, the observed $\Delta E_{\mathrm{int}}^{\mathrm{H} 9 \mathrm{H} 16}=-3.8 \mathrm{kcal} \mathrm{mol}^{-1}$ and $\Delta \sum_{\mathrm{X} \neq \mathrm{H} 9} E_{\mathrm{int}}^{\mathrm{H} 9 \mathrm{X}}=-3.9 \mathrm{kcal} \mathrm{mol}^{-1}$ might be seen as driving the twisted (lowest energy) conformer to the planar form of $\mathbf{b p h}$.

Hence, if the H9--H16 contact is not responsible for the higher energy of the planar bph from the FAMSEC perspectives, then what is? To pursue the source of planar bph instability we decided to apply the same protocol to C-atoms of the bay. This is because (i) each C-atom of the link (C3 and $\mathrm{C} 12)$ was shown here to be involved in most unfavorable change in the interaction energy with remaining atoms, the $\sum_{\mathrm{X} \neq \mathrm{C} 3} E_{\mathrm{int}}^{\mathrm{C} 3 \mathrm{X}}$ term, and (ii) these atoms were indicated previously $[40,41]$ as primarily responsible for the lower stability of the planar form of $\mathbf{b p h}$. It has also been reported [42] that the largest increase in QTAIM-defined atomic energies, by $22 \mathrm{kcal} \mathrm{mol}^{-1}$ at the HF/6-311++G(d,p) level, was obtained for the C-atoms linking the two rings (we obtained $21.6 \mathrm{kcal} \mathrm{mol}^{-1}$ at MP2). Moreover, most stabilized, by $-8.2 \mathrm{kcal} \mathrm{mol}^{-1}$ at $\mathrm{HF}$, were $\mathrm{H}$-atoms of the bay (we obtained $-7.8 \mathrm{kcal} \mathrm{mol}^{-1}$ at MP2). The analysis of changes in Bader's atomic energies of all atoms in bph [41], when going from ref to fin, lead to the conclusion (in 
agreement with the earlier report [40]) that the increase in the energy of C-atoms of the link overwrites the decrease in the atomic energies of $\mathrm{H}$-atoms involved in the clash. Furthermore, a very recent report [43] pointed at the $\mathrm{C}$-atoms to which ortho-hydrogens are bonded to as the main cause of destabilization of the planar form of $\mathbf{b p h}$.

We decided to select additional molecular fragments $\mathscr{G}$ made of atoms of the bay, $\{\mathrm{C} 3, \mathrm{C} 12\}$ and $\{\mathrm{C} 4, \mathrm{C} 13\}$, and applied the FAMSEC-based approach. Obtained relevant data for these fragments as well as $\mathscr{G}=\{\mathrm{H} 9, \mathrm{H} 16\}$ (the latter for convenience and to facilitate comparison) are included in Table 9. Focusing on the H9•••H16 interaction, it is seen that, in general, opposite in sign changes in these energy terms, when compared with those observed for the fragments made of C-atoms of the bay, are taking place. Most importantly, the $E_{\text {attr-mol }}^{\mathscr{E}}$ values of +3.2 and +2.5 $\mathrm{kcal} \mathrm{mol}^{-1}$ obtained for $\{\mathrm{C} 3, \mathrm{C} 12\}$ and $\{\mathrm{C} 4, \mathrm{C} 13\}$, respectively, clearly show that these fragments destabilize the planar form of $\mathbf{b p h}$. In the case of $\{\mathrm{C} 3, \mathrm{C} 12\}$, large and positive values of these C-atoms interactions with remaining atoms of $\mathbf{b p h}, E_{\text {int }}^{\text {EFF }}=9.1 \mathrm{kcal} \mathrm{mol}^{-1}$, in combination with somewhat repulsive interaction between them $\left(\Delta E_{\mathrm{int}}^{\mathrm{AB}}=1.1 \mathrm{kcal} \mathrm{mol}^{-1}\right)$ adds significantly to instability of the planar form. Note that the $E_{\text {int }}^{\mathscr{E} \not}$ energy term overwrites a significant decrease in $\Delta E_{\text {self }}^{\mathscr{E}}$ and interestingly, because $\Delta E_{\text {self }}^{\mathscr{E}}=-7 \mathrm{kcal} \mathrm{mol}^{-1}$ dominates $\Delta E_{\mathrm{int}}^{\mathrm{AB}}$ of $1.1 \mathrm{kcal} \mathrm{mol}^{-1}$, one observes locally stabilizing contribution of this fragment, $E_{\text {attr-loc }}^{\mathscr{E}}=-5.9 \mathrm{kcal} \mathrm{mol}^{-1}$. This overall effect can be seen as a competition between this fragment effective (destabilizing) and net (stabilizing) energy. This finding correlates well with a decrease in $E_{\text {add }}^{\mathrm{A}}$ of these atoms (see Table 2) which was also reported recently from the IQA and Hirshfeld atomic energy partitioning methods at the B3LYP level [42].

Quite a different picture emerges from analysis of the data obtained for the $\mathscr{I}_{=}=\{\mathrm{C} 4, \mathrm{C} 13\}$ fragment for which (i) 'small' but still unfavorable $\Delta E_{\mathrm{int}}^{\mathcal{E F}}$ is observed and (ii) the change in the self-atomic energies appears to be mainly responsible for destabilizing contributions coming 
from the $E_{\text {attr-mol }}^{\mathscr{E}}$ and $E_{\text {attr-loc }}^{\mathscr{E}}$ terms; in this case both changes, in the fragment's effective and net energies, are unfavorable.

\section{Table 9}

Energy components used for the interpretation of indicated molecular fragments in $\mathbf{b p h}$. $^{\mathrm{a}}$

\begin{tabular}{|c|c|c|c|}
\hline \multirow{2}{*}{ energy term } & \multicolumn{3}{|c|}{ molecular fragment $\mathscr{G}$} \\
\hline & $\{\mathrm{H} 9, \mathrm{H} 16\}$ & $\{\mathrm{C} 3, \mathrm{C} 12\}$ & $\{\mathrm{C} 4, \mathrm{C} 13\}$ \\
\hline$E_{\text {attr-mol }}^{\mathscr{E}}$ & -0.7 & 3.2 & 2.5 \\
\hline$\Delta E_{\text {int }}^{\mathrm{AB}}$ & -3.8 & 1.1 & 0.1 \\
\hline$\Delta \sum_{\mathrm{X} \neq \mathrm{A}} E_{\mathrm{int}}^{\mathrm{AX}}$ & -3.9 & 5.6 & 0.3 \\
\hline$\Delta E_{\mathrm{int}}^{\mathrm{A} \mathscr{H}}$ & -0.1 & 4.5 & 0.3 \\
\hline$\Delta E_{\mathrm{int}}^{\mathscr{E} \mathscr{F}}$ & -0.2 & 9.1 & 0.5 \\
\hline$\Delta E_{\text {self }}^{\mathscr{E}}$ & 3.3 & -7.0 & 1.9 \\
\hline$\Delta E_{\mathrm{add}}^{\mathscr{G}}$ & -0.6 & -1.4 & 2.2 \\
\hline$E_{\text {attr-loc }}^{\mathscr{E}}$ & -0.5 & -5.9 & 2.0 \\
\hline
\end{tabular}

Hence, our results partly agree with the earlier reports [40-42] in that either only C-atoms of the bay [40,41] or only $\mathrm{C}$-atoms to which ortho-H are bonded to [43] were found as main contributors to instability of planar bph. The apparent difference in conclusions arrived at [4042] can be attributed to the fact that these interpretations were mainly based on C-atoms atomic energies, virial [40,41] and IQA/FOHI [42] (FOHI stands for fractional occupation iterative Hirshfeld [43]). However, the FAMSEC-based interpretation, which involves all atomic energies in combination with all diatomic interactions throughout entire molecule, revealed that all C-atoms of the bay contribute (through different energy components) unfavorably to molecular energy of planar bph.

It is a well-known fact that the diatomic distance $\mathrm{d}(\mathrm{C} 3, \mathrm{C} 12)$ is larger in the planar bph (we obtained $\Delta \mathrm{d}(\mathrm{C} 3, \mathrm{C} 12)=0.012 \AA$ at MP2). Obviously, one can speculate that this is the natural 
result of accommodating clashing $\mathrm{H}$-atoms and this can be seen as a simple and reasonable argument which also agrees with a chemists' intuition. However, as has been shown in this work, the increase in energy of the forced-to-be planar form is not coming from the energy contributions made by ortho-hydrogens. Even though ortho-H atoms found themselves in the steric clash position in planar bph, their physical properties do not follow trends found for Oatoms in eclipsed form of $\mathbf{g c}$ but rather mimic those found for stabilized heteroatoms of Hen and Hea. To support this and for convenience, relevant energy terms are placed in Table 10. Although some energy term in Table 10 were discussed in details in previous sections, combining them together provides a compelling evidence that the $\mathrm{H}--\mathrm{H}$ contacts in planar bph show (i) many characteristics of a bonding interaction; notice that all parameters related to the classical intramolecular H-bonding in Hen and Hea agree entirely with those obtained for orthohydrogens whereas (ii) not a single parameter found for atoms of the $\{07,09\}$ fragment correlates well with relevant data found for ortho-hydrogens, exactly opposite trends are seen.

\section{Table 10}

Comparison of energy components obtained for indicated atoms. ${ }^{\mathrm{a}}$

\begin{tabular}{|c|c|c|c|c|c|c|c|c|c|}
\hline atom A & $\Delta E_{\text {add }}^{\mathrm{A}}$ & $\Delta E_{\mathrm{self}}^{\mathrm{A}}$ & $\Delta E_{\mathrm{int}}^{\mathrm{AB}}$ & $\Delta T^{\mathrm{X}}$ & $\Delta V_{\mathrm{ne}}^{\mathrm{XX}}$ & $\Delta V_{\mathrm{ee}}^{\mathrm{XX}}$ & $\Delta N^{\mathrm{X}}$ & $E_{\text {attr-mol }}^{\mathscr{E}}$ & $E_{\text {attr-loc }}^{\mathcal{E}}$ \\
\hline O7 (gc) & 9.6 & -0.3 & 25.8 & -1.8 & 44.3 & -42.8 & -0.019 & 13.1 & 25.3 \\
\hline H9 (bph) & -0.3 & 1.7 & -3.8 & 7.8 & -10.8 & 4.7 & 0.011 & -0.7 & -0.5 \\
\hline N7 (Hen) & -19.2 & 13.3 & -54.7 & 16.01 & -84.89 & 82.18 & 0.021 & -10.8 & -32.9 \\
\hline O7 (Hea) & -12.4 & 6.1 & -37.8 & 6.54 & -45.32 & 44.84 & 0.012 & -2.3 & -28.0 \\
\hline
\end{tabular}

a are the molecular fragments indicated in Table 1. All values in $\mathrm{kcal} \mathrm{mol}^{-1}$.

As this work has clearly evidenced, the property of an interaction and its strength strongly depend on the environment in which such an interaction occurs. Hence, even though stabilizing energy contribution made to a molecule by the $\mathrm{CH} \bullet \bullet \cdot \mathrm{HC}$ interaction was demonstrated, it does not imply that all such interactions, when in different molecules, will exhibit exactly the same characteristics. It is reasonable to assume that many might indeed destabilize a molecule even when $\Delta E_{\mathrm{int}}^{\mathrm{AB}}<0$; clearly each case deserves an in depth investigation and interpretation. 
Finally, three expressions for the mol-FAMSEC term are proposed here and it was of interest and importance to compare them because (i) different energy terms are used to compute fragment attributed changes in the energy of a molecular system and (ii) time required to compute the mol-FAMSEC term defined in Eq. 7 can easily be orders of magnitude larger when compared with time needed in the case of making use of Eqs. 14 and 17. For that purpose we used data obtained for Hen, Hea and gc because a full IQA analysis at the MP2 level was performed on these molecules. We found that the mol-FAMSEC terms computed from Eqs. 14 and 17 differed only by a fraction of $\mathrm{kcal} \mathrm{mol}^{-1}$ from that obtained from Eq. 7 . We obtained differences, (Eq. 14 - Eq. 7) and (Eq. 17 - Eq. 7), of 0.04, 0.22 and $-0.28 \mathrm{kcal} \mathrm{mol}^{-1}$ for Hen, Hea and gc, respectively. These 'small' differences are entirely caused by different sources used to compute interaction energy terms. Just as an example, when individual interaction energies were used to compute the interfragment energy term $E_{\text {int }}^{\mathscr{E} \not}$ for Hea we obtained $25.52 \mathrm{kcal} \mathrm{mol}^{-1}$ whereas the same energy contribution computed from Eq. 17 (where provided in AIMAll software $\sum_{\mathrm{X} \neq \mathrm{A}} 0.5 E_{\mathrm{int}}^{\mathrm{AX}}$ energy terms are used) resulted in $25.75 \mathrm{kcal} \mathrm{mol}^{-1}$ (a difference of 0.22 $\mathrm{kcal} \mathrm{mol}^{-1}$ ). Furthermore, we traced the origin of the observed $0.22 \mathrm{kcal} \mathrm{mol}^{-1}$ difference and established that 0.16 and $0.06 \mathrm{kcal} \mathrm{mol}^{-1}$ came from interactions involving $\mathrm{O} 7$ and $\mathrm{H} 12$ with remaining atoms in a molecule, respectively. In general, these results indicate that all three molFAMSEC terms are fully comparable and this confirms that there is no absolute need to compute all the possible diatomic interaction energies making applicability of the proposed protocol feasible for large(r) molecular systems.

\section{Conclusions}

The interaction energy between $\mathrm{A}$ and $\mathrm{B}$ atoms, $E_{\mathrm{int}}^{\mathrm{AB}}$, involved in an intramolecular interaction can be computed, e.g., by the use of the IQA energy and charge decomposition method. Although useful, this information is not sufficient to explain whether the interaction of interest, even when attractive in nature, $E_{\mathrm{int}}^{\mathrm{AB}}<0$, is (de)stabilizing a molecule as a whole. Two 
fragment attributed molecular system energy change (FAMSEC) terms: to the entire molecule, $E_{\mathrm{attr}-\mathrm{mol}}^{\mathscr{E}}=\Delta E_{\mathrm{eff}}^{\mathscr{E}}$, and localized to the fragment, $E_{\mathrm{attr}-\mathrm{loc}}^{\mathscr{E}}=\Delta E_{\mathrm{net}}^{\mathscr{E}}$ (where $E_{\mathrm{eff}}^{\mathscr{E}}$ and $\Delta E_{\mathrm{net}}^{\mathscr{E}}$ stand for the IQA-defined effective and net, respectively, energy of a molecular fragment) are proposed here. These atoms are treated as components of a selected molecular fragment $\mathscr{L}_{\text {whereas }}$ the remaining atoms of a molecule are seen as an additional molecular fragment $\mathscr{H}$. FAMSEC is an IQA-entrenched concept; hence, it accounts for changes in (i) one-body (self-atomic) and (ii) two-body (interaction energy between $\mathrm{A}$ and $\mathrm{B}$ as well as all interactions these two atoms are involved in with the atoms of the fragment $\mathscr{\not}$ ) molecular energy components when a molecular system undergoes a transformation from an arbitrarily selected reference (ref) state to its final (fin) structure where the $\mathrm{A} \bullet \bullet B$ interaction takes place. When a molecular system changes from ref to fin then the geometry of atoms $\mathrm{X} \in \mathscr{\Psi}$ can also change resulting in somewhat different density and charge distributions. In the proposed protocol, the geometric deformation energy of atoms $\mathrm{X} \in \mathscr{\Psi}$ is, although indirectly, accounted for to some extent. This is because these two changes in the atoms' physical properties, in addition to their different 3D placement in ref and fin states, must also contribute to the computed change in the interaction energy between atoms of the two fragments, $\mathscr{G}$ and $\mathscr{\Psi}$.

The nature of classical (i) stabilizing $\mathrm{N} \bullet \bullet \mathrm{H}$ and $\mathrm{O} \bullet \bullet \mathrm{H}$ interactions in the protonated forms of ethylenediamine (Hen) and ethanolamine (Hea), respectively, and (ii) destabilizing O--O steric contact in the eclipsed form of glycol (gc) was fully recovered. Focusing on the $\mathscr{G}$ fragments of the $\mathrm{N} \bullet \bullet \mathrm{H}$ and $\mathrm{O} \bullet \bullet \mathrm{H}$ interactions, their local $\left(E_{\text {attr-loc }}^{\mathscr{E}}\right)$ and on a molecular scale $\left(E_{\text {attr-mol }}^{\mathscr{E}}\right)$ energy contribution were stabilizing the respective molecules but exactly opposite trend was found for the $\mathscr{E}=\{\mathrm{O}, \mathrm{O}\}$ fragment in gc. Moreover, the origin of these interactions was explored. For each individual atom in $\mathrm{N} \bullet \bullet \mathrm{H}$ and $\mathrm{O} \bullet \bullet \mathrm{H}$, the additive atomic energy decreased due to favorable interactions with remaining atoms but self-atomic energies increased, as expected, on involvement in attractive and highly stabilizing intramolecular H-bonding; however, exactly 
opposite trends were found again for the O-atoms in gc. Data obtained also suggest that the $\mathrm{NH} \bullet \mathrm{H}$ in Hen, $\mathrm{NH} \bullet \mathrm{O}$ in Hea and $\mathrm{O} \bullet \cdot \mathrm{O}$ in ge might be seen as leading interactions which drive a molecule to the lowest energy state. In general, we have obtained a fully consistent description of these interactions and, importantly, it entirely agrees with chemists' intuition and knowledge.

Finally, using the same methodology, we investigated the planar and twisted conformers of biphenyl, bph. Results showed that the higher energy of the planar bph can be attributed to all C-atoms of a bay but the origin of their contributions is different. Unfavorable changes in interactions between the molecular fragment $\mathscr{G}_{2}=\{\mathrm{C} 3, \mathrm{C} 12\}(\mathrm{C}$-atoms linking the rings) and remaining atoms of $\mathbf{b p h}$ were found responsible for $E_{\text {attr-mol }}^{\mathscr{E}}>0$. Furthermore, destabilizing and comparable in value $E_{\text {attr-mol }}^{\mathscr{E}}$ term was also found for the remaining C-atoms of the bay, $\mathscr{E}=$ $\{\mathrm{C} 4, \mathrm{C} 13\}$, but the main contribution came from these atoms' self-atomic energies. On the other hand, considering the $\mathscr{G}=\{\mathrm{H}, \mathrm{H}\}$ fragment consisting of ortho-hydrogens, we have established that different energy contributions made by this fragment (i) do not conform to steric clash as observed in the case of $\mathrm{O}$-atoms in the eclipsed gc, (ii) resemble stabilizing interactions in Hen and Hea and (iii) cannot be linked with an increase of energy of the bph when in the planar form. Moreover, changes in physical properties of ortho-hydrogens and heteroatoms in Hen and Hea follow exactly the same trends on the intramolecular interaction formation whereas exactly opposite trends were found for O-atoms in the case of the O--O contact formation. Furthermore, we have found that (i) the $\mathrm{H} \bullet \bullet \mathrm{H}$ interaction is attractive in in both, twisted and planar, forms of bph and significantly stronger in the higher energy conformer $\left(\Delta E_{\mathrm{int}}^{\mathrm{H} 9 \mathrm{H} 16}=-3.8 \mathrm{kcal} \mathrm{mol}^{-1}\right.$ at MP2) and (ii) the sum of these atoms interactions with remaining atoms of planar bph (atoms of the molecular fragment $\mathscr{H})$ is small but attractive $\left(\Delta E_{\mathrm{int}}^{\mathrm{H} \mathscr{F}}=-0.1 \mathrm{kcal} \mathrm{mol}^{-1}\right.$ at MP2); hence, this clearly suggests that the $\mathrm{H}-\mathrm{-H}$ contacts do not drive a structural change from the planar to lower energy, twisted form of $\mathbf{b p h}$. 
Results obtained here clearly show that the FAMSEC terms are equally applicable to any kind of diatomic intramolecular interaction, regardless if they contribute in a (de)stabilizing manner and whether atoms involved are QTAIM or covalently (non)bonded. Also, although not investigated in this work, one should be able to (i) examine contributions made by polyatomic fragments of any size, e.g., functional groups and (ii) investigate intermolecular interactions; we hope to report our finding on such molecular systems soon.

We have demonstrated that the mol-FAMSEC term can also and conveniently be computed from derived expressions which require energy terms related only to the selected two atoms of the molecular fragment of interest. This makes computing of the derived mol-FAMSEC terms order(s) of magnitude less time demanding and investigation of interactions in large(r) molecules or molecular clusters feasible.

\section{Acknowledgments}

This work is based on the research supported in part by the National Research Foundation of South Africa (Grant No. 87777) and University of Pretoria. The author would also like to acknowledge Prof AM Pendás from University of Oviedo in Spain for his interesting and constructive comments on this work and ms.

\section{REFERENCES}

[1] G. Jeffrey, An Introduction to Hydrogen Bonding, Oxford University Press, New York, 1997.

[2] S.J. Grabowski, Hydrogen Bonding-New Insights. Vol. 3. Challenges and advances in computational chemistry and physics, J. Leszczynski, Ed.: Springer, 2006.

[3] K. Kitaura, K. Morokuma, A new energy decomposition scheme for molecular interactions within the Hartree-Fock approximation, Int. J. Quantum. Chem. 10 (1976) 325-340.

[4] M. Mitoraj, A. Michalak, Natural orbitals for chemical valence as descriptors of chemical bonding in transition metal complexes, J. Mol. Model. 13 (2007) 347-355.

[5] M. Mitoraj, A. Michalak, T. Ziegler, Combined charge and energy decomposition scheme for bond analysis, J. Chem. Theory Comput. 5 (2009) 962-975. 
[6] M.S. Gordon, J.H. Jensen, Understanding the hydrogen bond using quantum chemistry, Acc. Chem. Res. 29 (1996) 536-543.

[7] G. Buemi, F. Zuccarello, Is the intramolecular bond energy valuable from internal rotation barriers? J. Mol. Struct. (Theochem) 581 (2002) 71-85.

[8] L. Sobczyk, S.J. Grabowski, T.M. Krygowski, Interrelation between H-bond and Pielectron delocalization, Chem. Rev. 105 (2005) 3513-3560.

[9] S.J. Grabowski, Hydrogen bonding strength-measures based on geometric and topological parameters, J. Phys. Org. Chem. 17 (2004) 18-31.

[10] P. Lipkowski, A. Koll, A. Karpfen, P. Wolschann, An approach to estimate the energy of the intramolecular hydrogen bond, Chem. Phys. Lett. 360 (2002) 256-263.

[11] M. Jabłoński, G. Monaco, Different zeroes of interaction energies as the cause of opposite results on the stabilizing nature of $\mathrm{C}-\mathrm{H} \cdots \mathrm{O}$ intramolecular interactions, J. Chem. Inf. Model. 53 (2013) 1661-1675.

[12] M. Jabłoński, A. Kaczmarek, A.J. Sadlej, Estimates of the energy of intramolecular hydrogen bonds, J. Phys. Chem. A 110 (2006) 10890-10898.

[13] M. Jabłoński, Enaminoketony: teoretyczne badania dynamiki wewnątrzmolekularnej w układzie modelowym 3-aminoakroleiny, M.Sc. Dissertation, Nicolaus Copernicus University, Toruń, Poland, 2000.

[14] A. Nowroozi, H. Raissi, F. Farzad, The presentation of an approach for estimating the intramolecular hydrogen bond strength in conformational study of $\beta$-Aminoacrolein, $J$. Mol. Struct. (Theochem) 730 (2005) 161-169.

[15] E. Espinosa, E. Molins, C. Lecomte, Hydrogen bond strengths revealed by topological analyses of experimentally observed electron densities, Chem. Phys. Lett. 285 (1998) $170-173$.

[16] G. Gilli, F. Bellucci, V. Ferretti, V. Bertolasi, Evidence for resonance-assisted hydrogen bonding from crystal-structure correlations on the enol form of the $\beta$-diketone fragment, $\mathrm{J}$. Am. Chem. Soc. 111 (1989) 1023-1028.

[17] S.J. Grabowski, $\pi$-Electron delocalisation for intramolecular resonance assisted hydrogen bonds, J. Phys. Org. Chem. 16 (2003) 797-802.

[18] P. Gilli, V. Bertolasi, V. Ferretti, G. Gilli, Evidence for resonance-assisted hydrogen bonding. 4. Covalent nature of the strong homonuclear hydrogen bond. Study of the $\mathrm{O}-\mathrm{H}--$ O system by crystal structure correlation methods, J. Am. Chem. Soc. 116 (1994) 909-915.

[19] M.M Deshmukh, S.R. Gadre, L.J. Bartolotti, Estimation of intramolecular hydrogen bond energy via molecular tailoring approach, J. Phys. Chem. A 110 (2006) 12519-12523. 
[20] R.F.W. Bader, Atoms in Molecules: A Quantum Theory, Oxford University Press: Oxford, U.K., 1990.

[21] A.M. Pendás, E. Francisco, M.A. Blanco, C. Gatti, Bond paths as privileged exchange channels, Chem.-Eur. J. 13 (2007) 9362-9371.

[22] J. Cioslowski, S.T. Mixon, Topological properties of electron density in search of steric interactions in molecules: Electronic structure calculations on ortho-substituted biphenyls, J. Am. Chem. Soc. 114 (1992) 4382-4387.

[23] A. Haaland, D.J. Shorokhov, N.V. Tverdova, Topological analysis of electron densities: Is the presence of an atomic interaction line in an equilibrium geometry a sufficient condition for the existence of a chemical bond? Chem.-Eur. J. 10 (2004) 4416-4421.

[24] T. Strenalyuk, A. Haaland, Chemical bonding in the inclusion complex of $\mathrm{He}$ in adamantine (He@adam): The origin of the barrier to dissociation, Chem.-Eur. J. 14 (2008) 10223-10226.

[25] E. Cerpa, A. Krapp, R. Flores-Moreno, K.J. Donald, G. Merino, Influence of endohedral confinement on the electronic interaction between He atoms: $\mathrm{A} \mathrm{He} \mathrm{He}_{2} @ \mathrm{C}_{20} \mathrm{H}_{20}$ case study, Chem.-Eur. J. 15 (2009) 1985-1990.

[26] S. Grimme, C. Mück-Lichtenfeld, G. Erker, G. Kehr, H. Wang, H. Beckers, H. Willner, When do interacting atoms form a chemical bond? Spectroscopic measurements and theoretical analyses of dideuteriophenanthrene, Angew. Chem., Int. Ed. 48 (2009) 25922595 .

[27] P. Dem'yanov, P. Polestshuk, A bond path and an attractive Ehrenfest force do not necessarily indicate bonding interactions: Case study on $\mathrm{M}_{2} \mathrm{X}_{2}(\mathrm{M}=\mathrm{Li}, \mathrm{Na}, \mathrm{K} ; \mathrm{X}=\mathrm{H}, \mathrm{OH}$, F, Cl), Chem.-Eur. J. 18 (2012) 4982-4993.

[28] T. Tognettia, L. Joubert, On the physical role of exchange in the formation of an intramolecular bond path between two electronegative atoms, J. Chem. Phys. 138 (2013) 024102 .

[29] E.R. Johnson, S. Keinan, P. Mori-Sánchez, J. Contreras-García, A.J. Cohen, W. Yang, Revealing noncovalent interactions, J. Am. Chem. Soc. 132 (2010) 6498-6506.

[30] J. Contreras-García, E.R. Johnson, S. Keinan, R. Chaudret, J.P. Piquemal, D. Beratan, W. Yang, NCIPLOT: A program for plotting noncovalent interaction regions, J. Chem. Theory Comput. 7 (2011) 625-632.

[31] J. Contreras-García, W. Yang, E.R. Johnson, Analysis of hydrogen-bond interaction potentials from the electron density: Integration of noncovalent interaction regions, J. Phys. Chem. A 115 (2011) 12983-12990. 
[32] M.A. Blanco, A.M. Pendás, E. Francisco, Interacting quantum atoms: A correlated energy decomposition scheme based on the quantum theory of atoms in molecules, J. Chem. Theory Comput. 1 (2005) 1096-1109.

[33] E. Francisco, A.M. Pendás, M.A. Blanco, A molecular energy decomposition scheme for atoms in molecules, J. Chem. Theory Comput. 2 (2006) 90-102.

[34] Gaussian 09, Revision D.01, M.J. Frisch, G.W. Trucks, H.B. Schlegel, G.E. Scuseria, M.A. Robb, J.R. Cheeseman, G. Scalmani, V. Barone, B. Mennucci, G.A. Petersson, H. Nakatsuji, M. Caricato, X. Li, H.P. Hratchian, A.F. Izmaylov, J. Bloino, G. Zheng, J.L. Sonnenberg, M. Hada, M. Ehara, K. Toyota, R. Fukuda, J. Hasegawa, M. Ishida, T. Nakajima, Y. Honda, O. Kitao, H. Nakai, T. Vreven, J.A. Montgomery, Jr., J.E. Peralta, F. Ogliaro, M. Bearpark, J.J. Heyd, E. Brothers, K.N. Kudin, V.N. Staroverov, R. Kobayashi, J. Normand, K. Raghavachari, A. Rendell, J.C. Burant, S.S. Iyengar, J. Tomasi, M. Cossi, N. Rega, J.M. Millam, M. Klene, J.E. Knox, J.B. Cross, V. Bakken, C. Adamo, J. Jaramillo, R. Gomperts, R.E. Stratmann, O. Yazyev, A.J. Austin, R. Cammi, C. Pomelli, J.W. Ochterski, R.L. Martin, K. Morokuma, V.G. Zakrzewski, G.A. Voth, P. Salvador, J.J. Dannenberg, S. Dapprich, A.D. Daniels, Ö. Farkas, J.B. Foresman, J.V. Ortiz, J. Cioslowski, D.J. Fox, Gaussian, Inc., Wallingford CT, 2013.

[35] AIMAll (Version 13.11.04), T.A. Keith, TK Gristmill Software, Overland Parks KS, USA, 2013 (http://aim.tkgristmill.com).

[36] A.M. Pendás, E. Francisco, M.A. Blanco, M. A. Charge transfer, chemical potentials, and the nature of functional groups: answers from quantum chemical topology, Faraday Discuss. 135 (2007) 423-438.

[37] R. McWeeny, Methods of Molecular Quantum Mechanics, $2^{\text {nd }}$ ed., Academic Press, London, 1992.

[38] U. Koch, P.L.A. Popelier, Characterization of C-H-O Hydrogen Bonds on the Basis of the Charge Density, J. Phys. Chem. 99 (1995) 9747-9754.

[39] A.M. Pendás, M.A. Blanco, E. Francisco, The nature of the hydrogen bond: A synthesis from the interacting quantum atoms picture, J. Chem. Phys. 125 (2006) 184112.

[40] C.F. Matta, J. Hernández-Trujillo, T.H. Tang, R.F.W. Bader, Hydrogen-hydrogen bonding: A stabilizing interaction in molecules and crystals, Chem. Eur. J. 9 (2003) 19401951.

[41] J. Hernandez-Trujillo, C.F. Matta, Hydrogen-hydrogen bonding in biphenyl revisited, Struct. Chem. 18 (2007) 849-857. 
[42] K. Eskandari, C. Van Alsenoy, Hydrogen-hydrogen interaction in planar biphenyl: A theoretical study based on the interacting quantum atoms and Hirshfeld atomic energy partitioning methods, J. Comp. Chem. 35 (2014) 1883-1889.

[43] D. Geldof, A. Krishtal, F. Blockhuys, C. Van Alsenoy, An extension of the Hirshfeld method to open shell systems using fractional occupations, J. Chem. Theory Comput. 7 (2011) 1328-1335. 